\title{
Erfahrungen zur Gewalt gegen Rettungskräfte - aus der Sicht des DRK
}

\section{Experiences of Violence Against Emergency Ambulance Service Staff from the Perspective of the GRC}

Autoren

Peter Sefrin ${ }^{1}$, Annette Händlmeyer ${ }^{1}$, Thomas Stadler ${ }^{2}$, Wolfgang Kast ${ }^{1}$

Institute

1 Deutsches Rotes Kreuz, Generalsekretariat, Berlin, Deutschland

2 Bayerisches Rotes Kreuz, Abteilung Rettungsdienst, Landesgeschäftstelle, München, Deutschland

Schlüsselwörter

Zunahme der Gewalt im Rettungsdienst, Gewaltarten, Verortung der Gewaltanwendung, Täter, Schadensfolgen

\section{Key words}

increase in violence in the emergency ambulance services, types of violence, location of the use of violence, perpetrators, consequential of violence

Bibliografie

Notarzt 2021; 37: S1-S19

DOI $10.1055 / a-1310-6763$

ISSN 2364-7450

(c) 2021. Thieme. All rights reserved.

Georg Thieme Verlag KG, Rüdigerstraße 14,

70469 Stuttgart, Germany

Korrespondenzadresse

Prof. Dr. med. Peter Sefrin

Bundesarzt des DRK

Carstennstraße 58, 12205 Berlin

Deutschland

Tel.: 030/85404275, 0171/1237285

Sefrin.peter@outlook.de

\section{ZUSAMMENFASSUNG}

Berichte über Gewalt gegen Rettungskräfte werden immer häufiger. Vor diesem Hintergrund sollten spezielle Faktoren eruiert werden. Im Jahr 2019 führte das Deutsche Rote Kreuz (DRK) eine Umfrage zum Thema Gewalt gegen Einsatzkräfte im Rettungsdienst durch. In insgesamt 425 Fragebogen berichteten Einsatzkräfte über mindestens eine Gewaltanwendung in den vergangenen 12 Monaten. Ziel der Befragung war nicht die Erfassung der konkreten Anzahl der Übergriffe, sondern das Gewinnen von Angaben zu den Rahmenbedingungen. Die betroffenen Rettungsdienstmitarbeiter ${ }^{1}$ waren zu 73,4\% männlich und überwiegend in der Altersgruppe der 18-29-Jährigen. Es waren sowohl Notfallsanitäter (29,4\%), Rettungsassistenten (14,8\%) und Rettungssanitäter (33,6\%) als auch weitere Rettungsdienstmitarbeiter betroffen. Die Übergriffe waren hauptsächlich verbal (40,3\%). Der Anteil der tätlichen Gewalt betrug 14,4\%. Die körperlichen Angriffe ereigneten sich meist seltener als 1-2-mal pro Monat (83,8\%), während die verbalen Angriffe oft häufiger erfolgten. Jeder 2. Angriff passierte im innerstädtischen Bereich (52,2\%), wobei sozial problematische Wohngebiete vordergründig waren. Die Verteilung ist jedoch abhängig von Orten mit verschiedenen Einwohnerzahlen. Am häufigsten fanden die Gewaltanwendungen abends und nachts statt, meist während der Versorgung des Patienten (56,7\%). Der Täter war in drei Viertel der Fälle der Patient selbst, jedoch zu gleichen Teilen auch Freunde (42,6\%) und Angehörige (40,7\%). Ein Schwerpunktort der Übergriffe sind unabhängig von der Größe der Orte Großveranstaltungen. Soweit feststellbar spielten Alkohol und Drogen eine wesentliche Rolle. Die Folge seitens der Betroffenen war bei 2,1\% eine so schwere Schädigung, dass sie zu einer Krankschreibung von 1 Tag und im Extremfall bis zu 40 Tagen führte. In jedem 2. Fall wurde die Polizei entweder aufgrund des Meldebildes oder durch den Rettungsdienst vor Ort alarmiert. 76\% der Betroffenen wünschen eine gesonderte Schulung im Umgang mit Gewalt im Rahmen der Fortbildung. Das Mittel der Wahl seitens der Politik zur Verbesserung der Situation ist nach Meinung der Rettungsdienstmitarbeiter die Anhebung der pekuniären Strafen. Eine weitere Strafverschärfung scheint vor dem Hintergrund des Täterprofils und der Umgebungsbedingungen und der bei der in der Bevölkerung teilweise bestehenden Respektlosigkeit kein gangbarer Weg zu sein. Inwieweit eine intensivere Aufklärung zu einer Verbesserung der Situation führen kann, kann derzeit nur vermutet werden. Aus den Ergebnissen abgeleitet wurden Forderungen für die Zukunft erhoben.

\footnotetext{
$1 \quad$ Im Sinne der besseren Lesbarkeit wird in diesem Beitrag ausschließlich die männliche Form verwendet. Die weibliche Form ist selbstverständlich immer mit eingeschlossen.
} 


\section{ABSTRACT}

Reports about violence against emergency ambulance service staff are becoming increasingly frequent. Against this backdrop, descriptive factors about the assaults should be identified. In 2019, the German Red Cross (GRC) conducted a survey on violence against employees in emergency medical services. In a total of 425 questionnaires, emergency personnel reported at least one instance of violence in the past 12 months. The aim of the survey was not to record the actual number of assaults but to provide information on the framework conditions. $73.4 \%$ of the affected ambulance service employees were male and mostly in the age group of 18-29 years. Emergency care paramedics (29.4\%), paramedics (14.8\%) and emergency medical technicians $(33.9 \%)$ as well as other emergency ambulance service employees were affected. $^{2}$ The assaults were mainly verbal $(40.3 \%)$. The percentage of physical violence was $14.4 \%$. While physical attacks often occurred less than 1.2 times per month (83.3\%), verbal attacks occurred more frequently. Every second attack

2 Erläuterung: "Emergency care paramedic" entspricht hier dem deutschen Begriff Notfallsanitäter. "Paramedic" entspricht hier dem deutschen Begriff Rettungsassistent. "Emergency medical technician” entspricht hier dem deutschen Begriff Rettungssanitäter. occurred in inner-city areas (52.2\%), with socially problematic residential areas being the most prominent. The distribution, however, also depends on the number of inhabitants of the places. The most frequent incidents of violence occurred in the evening and at night, mostly during the care of the patient $(56.7 \%)$. In three quarters of the cases, the perpetrator was the patient himself, but friends (42.6\%) and relatives (40.7\%) were also involved. The focal point of the assaults is independent of the scale of the events. As far as it can be ascertained, alcohol and drugs played a major role. The consequence for the affected was that $2.1 \%$ suffered such severe damage that they were on sick leave for 1 day $(22.2 \%)$ and in extreme cases up to 40 days. In every second case, the police was alerted either by the reported emergency case or by the emergency ambulance service on site. $76 \%$ of those affected would like to receive special training in dealing with violence as part of the educational program. The preferred approach on the part of politics to improve the situation is, according to the emergency ambulance service staff, the increase in pecuniary sanctions. A further increase in non-financial punishment does not appear to be a viable option, given the profile of the perpetrator, the environmental conditions and the disrespect that exists in some areas of the population. To what extent a more intensive education can lead to an improvement of this situation can currently only be speculated. Requirements for the future were formulated based on the results.

\section{Einleitung}

Gewalt bei der Ausübung des (Rettungs-)Dienstes ist ein ernst zu nehmendes Problem. Das Thema Gewalt gegen Repräsentanten der öffentlichen Ordnung ist in den Medien präsent und wird häufig bezogen auf Einzelpersonen. Primär konzentrierte sich dies auf den Personenkreis der Polizei und führte in der Folge zu einer Ausweitung und Verschärfung der $\S \S 113$ und 114 StGB (Widerstand gegen Vollstreckungsbeamte und Personen, die Vollstreckungsbeamten gleichstehen). Inzwischen hat sich jedoch der Fokus der Gewalt auch auf Rettungskräfte und Feuerwehrpersonal erweitert. Die Übergriffsformen sind vielfältig. Es gibt immer mehr Menschen, die Mitarbeiter des Rettungsdienstes als Vertreter einer staatlichen Institution ansehen, die sie nicht akzeptieren und als Feind betrachten. Das Rettungsdienstpersonal kann nicht mehr darauf vertrauen, aufgrund seiner Dienstkleidung vor Gefahren geschützt zu sein [1]. Es wird berichtet, dass Einsatzkräfte des Rettungsdienstes bei ihren Einsätzen behindert und angegriffen werden. In den letzten 5 Jahren wird ein stetiger Anstieg der Bedrohungszahlen konstatiert [2]. Das baden-württembergische Innenministerium teilte mit, dass im Vergleich zum Jahr 2011 Rettungs- und Feuerwehrkräfte mindestens 3-mal häufiger attackiert werden. Verglichen mit dem Jahr 2017 sei die Zahl der körperlichen Angriffe im Jahr 2019 um mehr als ein Drittel gestiegen. 2019 wurden 190 Fälle von Gewalt gegen Rettungskräfte registriert, im Jahr zuvor waren es noch 139 [3]. Während die Polizei als Repräsentant der „Staatsmacht“ als Angriffsziel noch theo- retisch nachvollziehbar wäre, ist die Ausdehnung von Gewalt gegen „Helfende“ nicht primär einsichtig. Schlagworte wie „Vom Helden zum Prügelknaben“ (Stuttgarter Nachrichten vom 04.01.2018) machen die Runde. Es fehlen allerdings exakte, regional übergreifende Zahlen über Anzahl und Art der Gewaltattacken sowie deren Auswirkungen gegenüber Mitarbeiter des Rettungsdienstes. Bei der offiziellen Statistik (z. B. Polizeiliche Kriminalstatistik) wird nicht isoliert auf Rettungskräfte eingegangen, sondern diese neben den Polizeikräften als „andere Einsatzkräfte“ subsumiert. Um das Ausmaß der resultierenden Probleme zu quantifizieren, hat das Deutsche Rote Kreuz (DRK) eine bundesweite Umfrage unter Mitarbeitern des Rettungsdienstes durchgeführt.

\section{Methodik}

Es handelt sich um eine deskriptive Auswertung der Daten eines Fragebogens, der ausschließlich an DRK-eigene Rettungsdienste verschickt und freiwillig und anonym ausgefüllt wurde. Die Daten wurden als Anzahl (n) und Anteil (\%) dargestellt. Die Datenauswertung der Gesamtheit der Fragebogen erfolgte mit MS Excel für Windows. Neben geschlossenen Fragen mit vorgegebenen Antwortmöglichkeiten ergänzten offene Fragen mit der Möglichkeit der Freitexteingabe den Erhebungsbogen. Die Daten bezogen sich retrospektiv auf einen Zeitraum von einem Jahr und wurden von August bis November 2019 gesammelt. Insgesamt handelt es sich demnach um eine zufällige Positivauswahl. In die Auswer- 
tung wurden nur Fragebogen aufgenommen, die vollständig ausgefüllt waren. Insgesamt konnten 425 Fragebogen ausgewertet werden. 14 Landesverbände beteiligten sich an der Fragebogenaktion, wobei die meisten Rückmeldungen aus Sachsen (27,1\%), gefolgt von Schleswig-Holstein (18,6\%) und Bayern (17,9\%), kamen.

In die Diskussion eingezogen wurde eine gesonderte regionale Auswertung des Rettungsdienstes in Bayern aus der Zeit zwischen 01.01. und 31.12.2019 mit 61 Meldungen.

\section{Definition und Formen der Gewalt [4]}

Unter Gewalt in diesem Zusammenhang werden Handlungen verstanden, die auf Menschen schädigend einwirken, wobei zwischen körperlicher und verbaler Gewalt unterschieden wird. Im juristischen Sprachgebrauch wird Gewalt definiert als eine zielgerichtete Einwirkung auf einen Menschen, die bei diesem zur physischen oder psychischen Schädigung führt [5].

Unter körperlicher Gewalt wird ein aggressives Verhalten verstanden, das zu Schädigungen und/oder Verletzung der körperlichen Unversehrtheit eines anderen führt. Hierzu gehören neben Androhung von Gewalt u. a. gezieltes Bespucken, Schubsen, Treten, Beißen, Würgen, Haareziehen.

Verbale Gewalt bedeutet, dass das Opfer durch Worte bedroht und/oder beleidigt wird [6]. Es handelt sich dabei um Kommunikationsformen, bei der die Sprache (oft auch in Kombination mit der Mimik und Gestik) genutzt wird, um den anderen gezielt zu beleidigen, zu demütigen, zu diskriminieren oder ihn auszugrenzen. In unserer Auswertung werden auch Beschimpfungen und Beleidigungen subsumiert, wobei darunter ein Angriff auf die Ehre des Mitarbeiters des Rettungsdienstes durch Kundgabe der Missachtung verstanden wird.

Eine Sonderform ist die nonverbale Gewalt, die Übergriffe umfasst, die durch Gesten zum Ausdruck gebracht werden und einen eindeutigen herabwürdigenden oder bedrohlichen Charakter haben (Gewaltandrohung, ,Stinkefinger“, geballte Faust u. a.)

Gewalt richtet sich nicht nur gegen Personen, sondern auch gegen Sachen mit der Folge der Sachbeschädigung (z. B. gegen Fahrzeuge oder deren Ausstattung) mitunter durch Zertreten von Ausrüstungsgegenständen, Abbrechen von Anbauten am Fahrzeug oder Beschmutzen. Im Extremfall zählt auch der Diebstahl von Ausstattungsteilen zur Sachbeschädigung.

Sexuelle Gewalt ist jede Art der körperlichen Belästigung einer anderen Person in sexueller Hinsicht in Form von Handlungen und/oder Androhung. Sie kann sowohl weibliche als auch männliche Opfer betreffen.

Waffengewalt ist eine der stärksten Gewaltanwendungen mit realen Waffen (Messer, Schuss- und Stichwaffen), aber auch Gewalt durch andere Gegenstände wie bspw. Hammer, Schraubenzieher, Flaschen, Glasscherben.

\section{Ergebnisse}

\section{Anzahl der Übergriffe}

Es war nicht vorgesehen, die konkrete Anzahl der einzelnen Übergriffe zu dokumentieren, sondern einen Überblick über die Art und die Folgen der Übergriffe aufzuzeichnen. Aus der $\bullet$ Tab. 1 wird deutlich, dass überschlagsmäßig in dem 1-Jahres-Zeitraum ca. mehr als 10000 Übergriffe stattfanden. Drei Mitarbeiter geben immerhin an, dass verbale Angriffe täglich erfolgen.

Auch wenn die Anzahl der körperlichen Übergriffe geringer war, so sind doch schätzungsweise ca. 3000 Übergriffe im Untersuchungszeitraum erfolgt. Mehr als erstaunlich ist die Aussage, dass inzwischen körperliche Übergriffe täglich bzw. in der Nachtschicht fast immer erfolgen ( $\triangleright$ Tab. 2).

\section{Betroffene}

Bei den Befragten handelt es sich um Rettungskräfte des DRK in Deutschland. In Analogie zu der geschlechtlichen Zusammensetzung des Personals im Rettungsdienst waren 73,4\% der Betroffenen männlichen und 26,4\% weiblichen Geschlechtes ( $\bullet$ Abb. 1). Die Betroffenen waren überwiegend in der Altersgruppe der 18bis 29-Jährigen (49,6\%), gefolgt von den 30 - bis 39-Jährigen $(23,8 \%)$ und den 40 - bis 49-Jährigen (16,5\%) ( A Abb. 2 ).

Die Rettungsdienstgesetze der Länder erlauben die Besetzung der Rettungsmittel mit Mitarbeitern mit verschiedenen Qualifikationen. Somit hatten auch die Betroffenen verschiedene Qualifikationsstufen vom Sanitäts- bzw. Rettungshelfer bis zum Notfallsanitäter ( $\bullet$ Tab. 3 ).

Somit erklärt sich auch, dass die Tätigkeit im Rettungsdienst und damit die Berufserfahrung sich über verschiedene Zeiträume spannten. Die meisten (36,5\%) hatten eine Berufserfahrung von bis zu 5 Jahren, gefolgt von 27,5 bzw. 26,4\% mit 5-15 Jahren und über 15 Jahren Berufserfahrung. 81,4\% waren hauptamtlich und 27,3\% ehrenamtlich im Rettungsdienst tätig. Dabei wird ersichtlich, dass einzelne Einsatzkräfte im Berichtszeitraum sowohl hauptamtlich als auch ehrenamtlich tätig waren.

- Tab. 1 Anzahl der verbalen Übergriffe

\section{verbale Übergriffe}

seltener als 1-2-mal pro Monat (0-11-mal pro Jahr)

1-2-mal pro Monat (12-24-mal pro Jahr)

1-2-mal pro Woche (52-104-mal pro Jahr)

mehr als 2-mal pro Woche (mehr als 104-mal pro Jahr)

täglich

keine Angabe

\begin{tabular}{|c|}
\hline ت̈ا \\
\hline $36,2 \%(n=154)$ \\
\hline $44,0 \%(n=187)$ \\
\hline $12,5 \%(n=53)$ \\
\hline $5,2 \%(n=22)$ \\
\hline $0,7 \%(n=3)$ \\
\hline $1,4 \%(n=6)$ \\
\hline
\end{tabular}




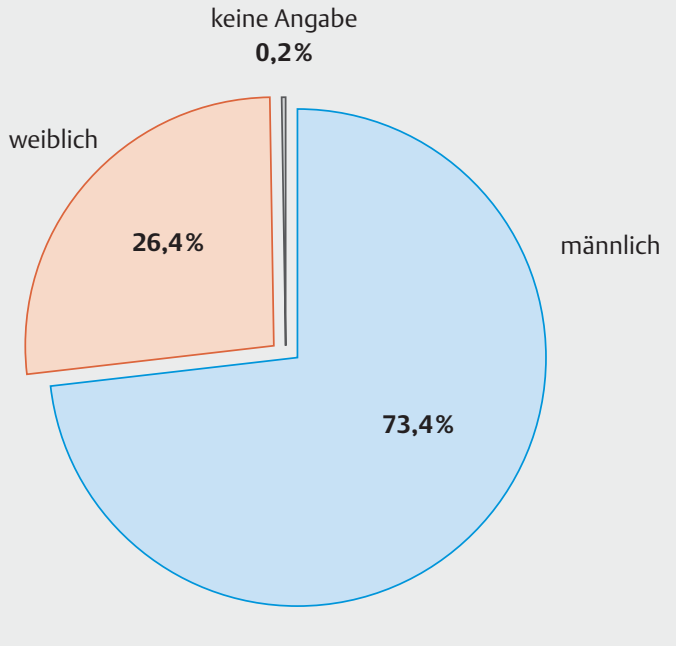

- Abb. 1 Geschlecht der Betroffenen.
Nachdem aus der Analyse nicht hervorgeht, in welchem zeitlichen Umfang die Betroffenen im Rettungsdienst eingesetzt waren, besteht bei längerem und wiederholtem Einsatz die Möglichkeit (oder Wahrscheinlichkeit), dass sie im Analysezeitraum mehrmals mit möglichen Gewaltanwendungen konfrontiert waren, was nachfolgend zu Mehrfachnennungen führen kann.

Nicht verwunderlich ist bei dieser Verteilung des Personals, dass auch die Gewaltereignisse sich nicht nur auf den Einsatz bestimmter Rettungsmittel beschränkten. Es waren Einsätze mit allen Fahrzeugtypen, wobei auch der Rettungshubschrauber als Rettungsmittel betroffen war. Am häufigsten war das Personal der Notfallrettungsmittel Ziel der Gewalt (75,3\%) ( $\triangleright$ Tab. 4).

Da sich die Gewaltanwendung teilweise nicht nur auf das Personal des Rettungsdienstes beschränkte, konnte festgestellt werden, dass in 42,8\% der Nennungen daneben auch andere Personen betroffen waren, was in $22,6 \%$ der Fälle sogar mehrmals der Fall war.

\section{Art der Gewalt bzw. Übergriffe}

Verbale An- bzw. Übergriffe waren mit einem Anteil von 40,3\% der angezeigten Übergriffe am häufigsten, wobei allerdings 5 verschiedene Formen berichtet wurden. Die häufigste Form waren Beschimpfungen und Beleidigungen mit 91,1\%, gefolgt von Androhung von Gewalt (55,3\%). Der Anteil der tätlichen Übergriffe betrug $14,4 \%$ bei 10 verschiedenen Formen. Die häufigste Form

- Tab. 2 Anzahl der körperlichen Übergriffe.

\author{
körperliche Übergriffe \\ seltener als 1-2-mal pro Monat (0-11-mal pro Jahr) \\ 1-2-mal pro Monat (12-24-mal pro Jahr) \\ 1-2-mal pro Woche (52-104 mal pro Jahr) \\ mehr als 2-mal pro Woche \\ täglich \\ in der Nachtschicht fast immer \\ keine Angabe
}

Fälle mit Übergriffen ( $n=425)$

$83,8 \%(n=356)$
$11,8 \%(n=50)$
$0,9 \%(n=4)$
$0,0 \%(n=0)$
$0,2 \%(n=1)$
$0,2 \%(n=1)$
$3,1 \%(n=13)$

-Tab.3 Qualifikation des Rettungspersonals.

\begin{tabular}{|l|l|}
\hline & Fälle mit Übergriffen (n= 425) \\
\hline Notfallsanitäter & $29,4 \%(n=125)$ \\
\hline Rettungsassistent & $14,8 \%(n=63)$ \\
\hline Rettungssanitäter & $33,6 \%(n=143)$ \\
\hline sonstige & $11,8 \%(n=50)$ \\
\hline davon u. a.: & Basis $n=50$ \\
\hline - Sanitätshelfer & $32,0 \%(n=16)$ \\
\hline - Azubi NFS & $22,0 \%(n=11)$ \\
\hline - Sanitäter & $20,0 \%(n=10)$ \\
\hline - Rettungshelfer & $8,0 \%(n=4)$ \\
\hline keine Angabe & $11,1 \%(n=44)$ \\
\hline
\end{tabular}




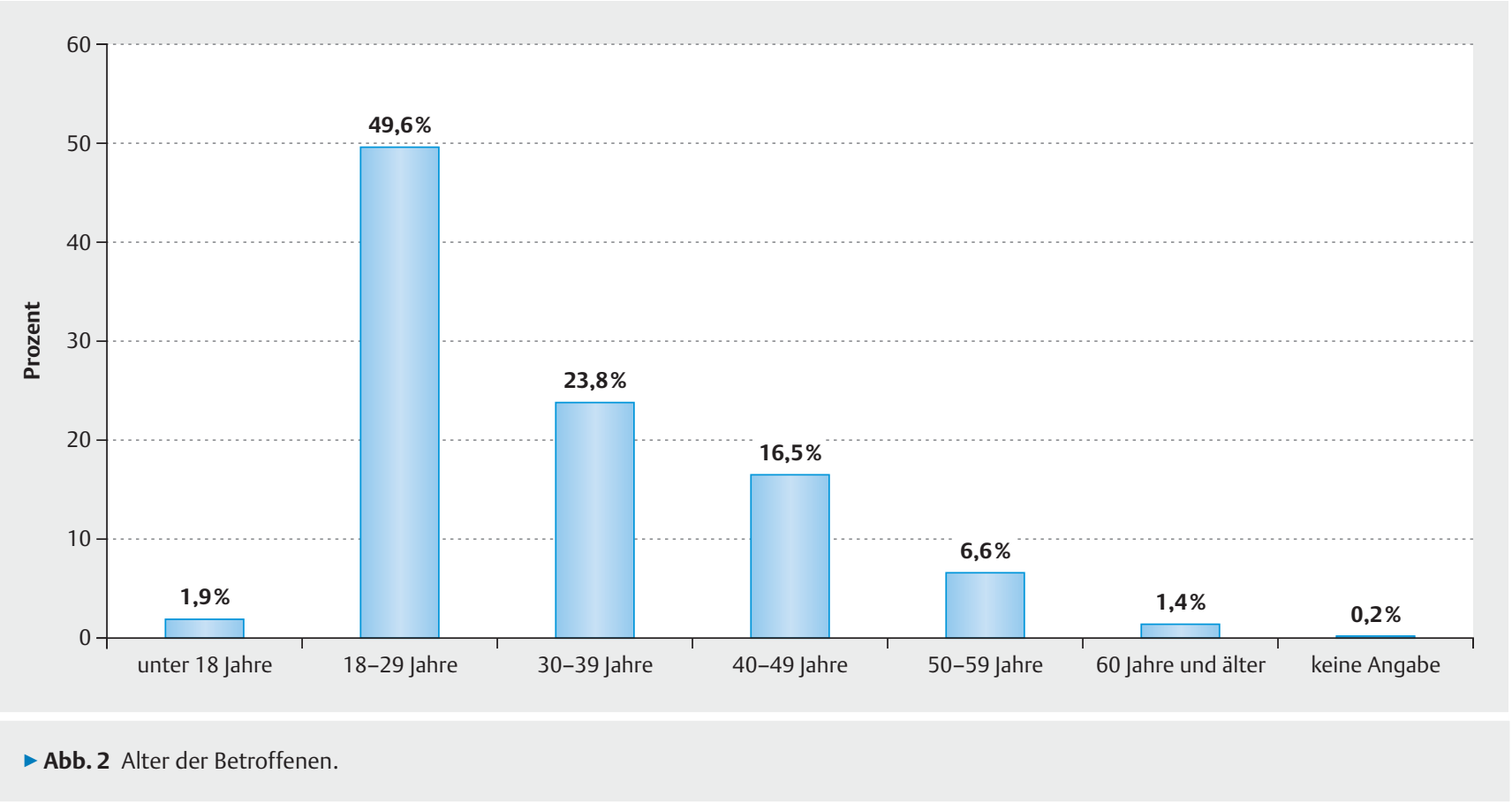

war Schlagen und Treten (32,7\%) gleichauf mit Schubsen (31,5\%). An 2. und 3. Stelle stehen Anspucken (28\%) und Bedrängen mit körperlichem Kontakt (28,5\%). Am seltensten waren konkrete Angriffe mit einem Gegenstand $(6,4 \%)$ und die Ausübung von Waffengewalt $(0,7 \%)$. Eine Sonderform der Gewaltanwendung ist die sexuelle Belästigung, die sowohl in verbaler (10,6\%) wie auch in körperlicher Form vorkam (3,1\%) ( A Abb. 3).

\section{Ort bzw. Situation der Gewaltanwendung}

Bei Einsätzen an sozialen Brennpunkten könnte plausiblerweise mit einer erhöhten Wahrscheinlichkeit für Auseinandersetzungen mit Gewaltanwendungen gerechnet werden, weshalb analysiert wurde, wo die jeweiligen Gewaltanwendungen gegen Rettungskräfte erfolgten. Es zeigte sich bei Mehrfachnennungen der Einsatzorte, dass die häufigsten Gewaltanwendungen bei Einsätzen im innerstädtischen Bereich (undifferenziert) erfolgten (52,2\%), gefolgt von Einsätzen in sozial problematischen Wohngebieten
(51,8\%). An 3. Stelle waren es Einsätze in bürgerlichen Wohngebieten (47,1\%) und bei Großveranstaltungen (47,1\%). Am Hauptbahnhof der verschiedenen Ortschaften und in „Kneipenvierteln" befanden sich weitere wesentliche Einsatzorte. Seltener waren Gewaltanwendung in Außerortsbereichen, Industriegebieten und Rotlichtvierteln ( $\triangleright$ Abb. 4 ).

Um zur Frage der Verortung Stellung nehmen zu können, wurde auch der Einsatzort nach der Anzahl der Einwohner aufgeschlüsselt, um erkennen zu können, ob sich ein Schwerpunkt im städtischen oder ländlichen Bereich darstellt. Deutlich zu erkennen ist hier eine Häufung im klein- und mittelstädtischen Bereich ( $\triangleright$ Abb. 5 ).

Bei der Zuordnung der Gewalttaten zu den differenten Verortungen konnten unterschiedliche Schwerpunkte des Geschehens ausgemacht werden. In Dörfern lag der Schwerpunkt in der „bürgerlichen“ Wohngegend (61,1\%) sowie bei den Großveranstaltungen (59,7\%). In den Kleinstädten lagen Innenstadt und

Tab. 4 Fahrzeuge, auf denen die Betroffenen eingesetzt waren (Mehrfachnennungen).

\begin{tabular}{|l|c|}
\hline & Fälle mit Übergriffen (n=425) \\
\hline NEF & $29,4 \%(n=125)$ \\
\hline RTW/NAW & $75,3 \%(n=320)$ \\
\hline KTW & $28,9 \%(n=123)$ \\
\hline ITW & $0,9 \%(n=4)$ \\
\hline RTH & $1,9 \%(n=8)$ \\
\hline sonstige & $10,4 \%(n=44)$ \\
\hline keine Angabe & $8,0 \%(n=34)$ \\
\hline
\end{tabular}




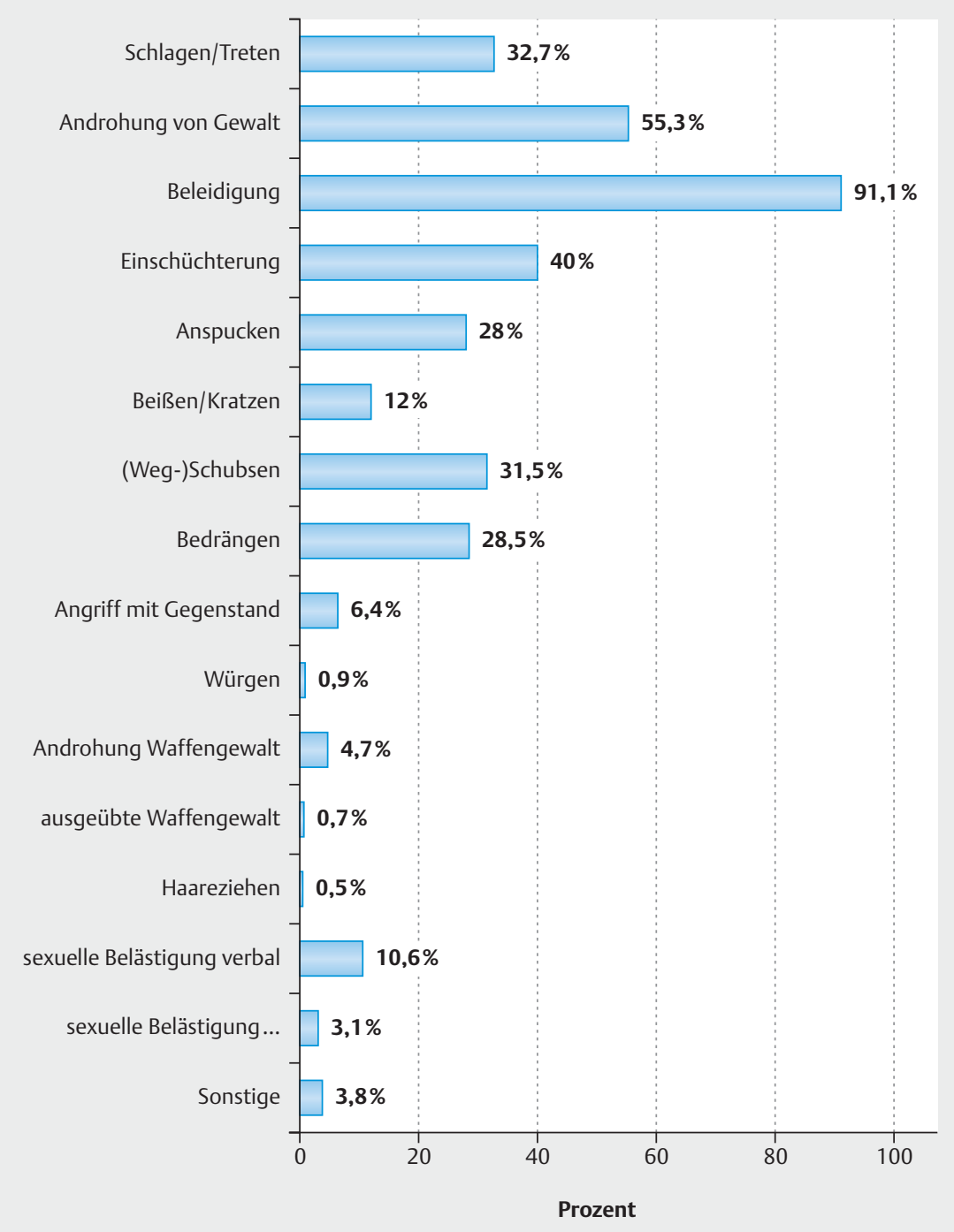

- Abb. 3 Art der Gewalt (Mehrfachnennungen).

bürgerliche Wohngegend gleich auf $(57,9 \%)$, aber auch hier waren Großveranstaltungen ein Schwerpunkt (54,5\%). In den Städten waren es die Innenstadt $(66,3 \%)$ und die sozial problematischen Wohngegenden (63,9\%), die einen Brennpunkt darstellten. Aber auch hier waren Großveranstaltungen mit einem Anteil von $56,2 \%$ beteiligt. In den Großstädten spielten sich die Gewaltanwendungen vordergründig in der Innenstadt (73,1\%) und in den Wohngegenden ab, wobei auch hier die sozial problematischen (70,1\%) gegenüber den bürgerlichen Wohngegenden $(67,2 \%)$ überwogen. In den großen Großstädten lag der Schwerpunkt bei Innenstadt und den Großveranstaltungen (jeweils $61,5 \%)$. Neben den sozial problematischen Wohngegenden spielte hier der Hauptbahnhof als sozialer Brennpunkt eine Rolle $(\triangleright$ Tab. 5).

Aus dieser Auswertung kann geschlossen werden, dass sich in den verschiedenen Regionen differente Schwerpunkte ergeben, wobei ersichtlich wird, dass Gewalttaten sich keineswegs nur in
Großstädten oder an sozialen Brennpunkten ereignen. Allen Verortungen gemeinsam sind Großveranstaltungen als eine wesentliche Lokalisation für Übergriffe gegen Rettungspersonal.

\section{Häufigkeit der Übergriffe}

Aufgrund der Häufigkeiten der verbalen (40,3\%) und der körperlichen/tätlichen Übergriffe (14,4\%) und der $13,6 \%$ sexuellen Übergriffe wurden die beiden erstgenannten einer genaueren Betrachtung unterzogen ( $\triangleright$ Abb. 6).

Es ist deutlich, dass die Häufigkeit der berichteten Gewaltanwendungen sich mehrfach im Bereich von 1-2-mal im Monat abspielte. Es kann demnach nicht für den einzelnen Mitarbeiter von einem singulären Ereignis im Rettungsdienst gesprochen werden. Verbale Übergriffe waren wesentlich häufiger mehrfach über einen Zeitraum von Wochen und Monate angefallen, während die körperlichen Übergriffe meist seltener in einem Zeitraum von 12 Monaten erfolgten. 


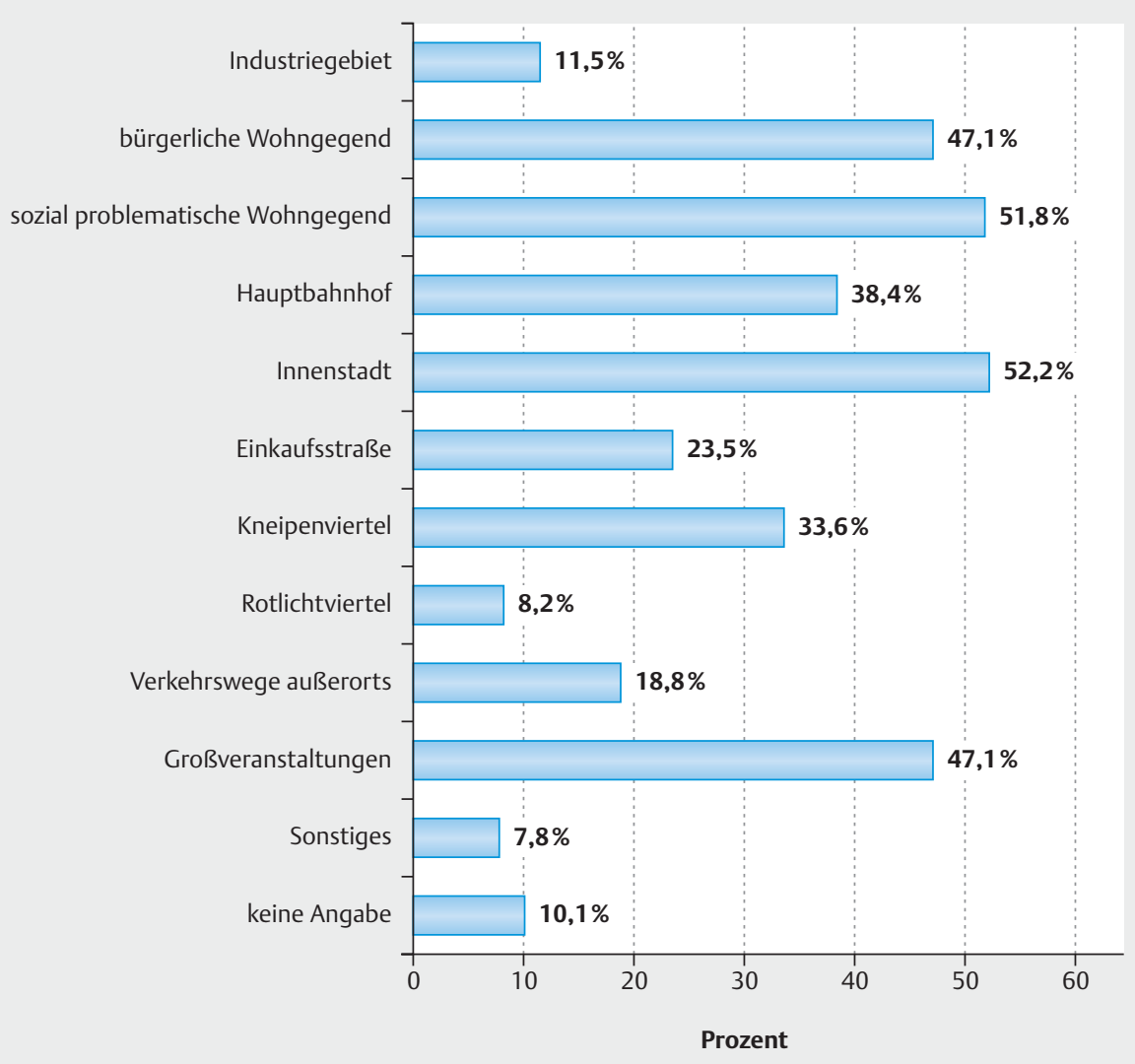

- Abb. 4 Einsatzumfeld (Mehrfachnennungen).

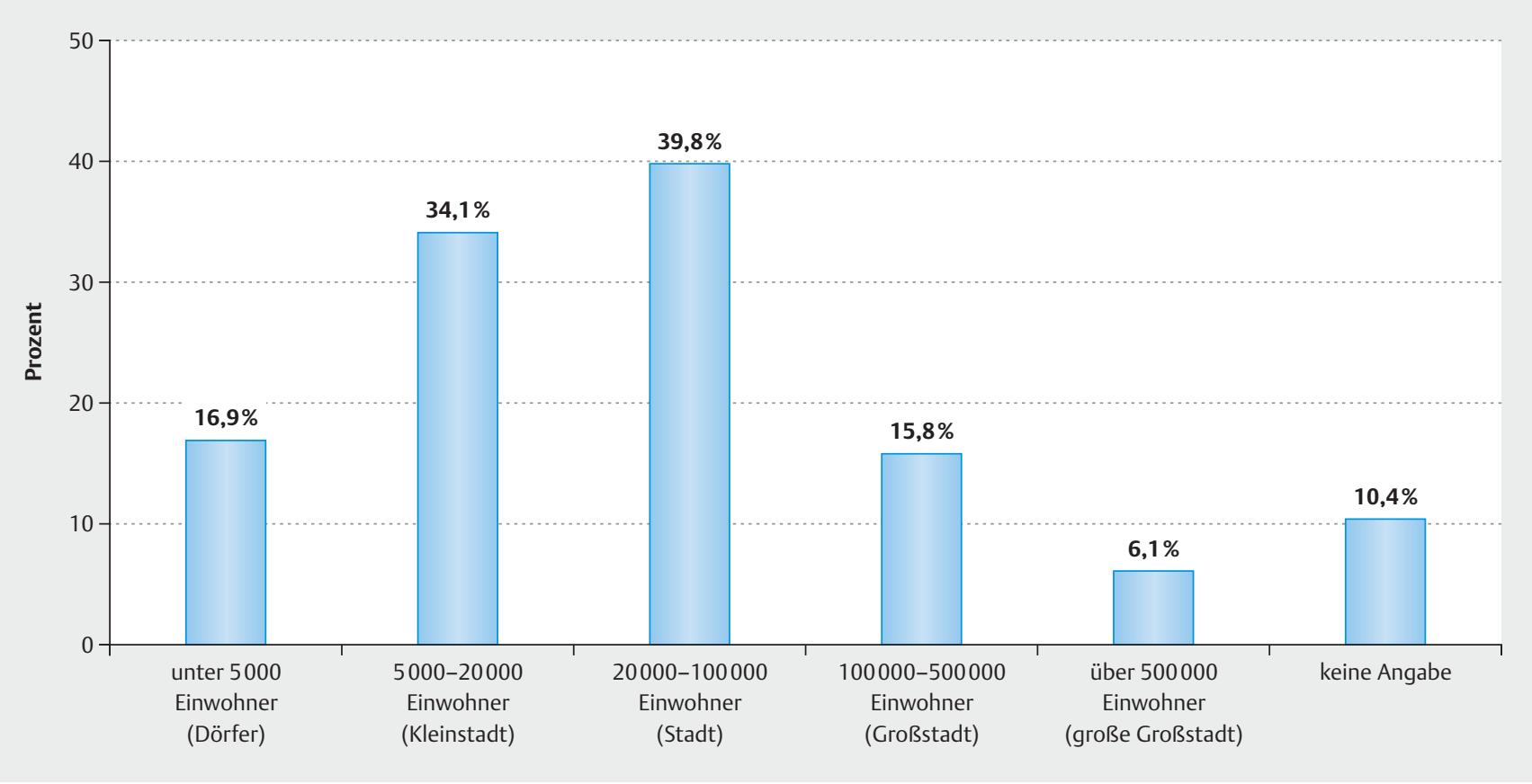

- Abb. 5 Orte, an denen die Gewaltanwendungen erfolgten (Mehrfachnennungen). 
\ Tab. 5 Einsatzort in Abhängigkeit von der Größe der Ortschaften entsprechend der Einwohnerzahl (Mehrfachnennungen).

\begin{tabular}{|c|c|c|c|c|c|}
\hline Einsatzumfeld & $\begin{array}{l}\text { bis } 5000 \\
(n=72)\end{array}$ & $\begin{array}{l}\text { über } 5000- \\
20000(n=145)\end{array}$ & $\begin{array}{l}\text { über } 20000- \\
100000(n=169)\end{array}$ & $\begin{array}{l}\text { über } 100000- \\
500000(n=67)\end{array}$ & $\begin{array}{l}\text { über } 500000 \\
(n=26)\end{array}$ \\
\hline Hauptbahnhof & $23,6 \%(n=17)$ & $33,1 \%(n=48)$ & $52,7 \%(n=89)$ & $67,2 \%(n=45)$ & $42,3 \%(n=11)$ \\
\hline Innenstadt & $40,3 \%(n=29)$ & $57,9 \%(n=84)$ & $66,3 \%(n=112)$ & $73,1 \%(n=49)$ & $61,5 \%(n=16)$ \\
\hline bürgerliche Wohngegend & $61,1 \%(n=44)$ & $57,9 \%(n=84)$ & $50,3 \%(n=85)$ & $55,2 \%(n=37)$ & $42,3 \%(n=11)$ \\
\hline $\begin{array}{l}\text { sozial problematische } \\
\text { Wohngegend }\end{array}$ & $47,2 \%(n=34)$ & $55,9 \%(n=81)$ & $63,9 \%(n=108)$ & $70,1 \%(n=47)$ & $57,7 \%(n=15)$ \\
\hline Einkaufsstraße & $23,6 \%(n=17)$ & $26,9 \%(n=39)$ & $29,6 \%(n=50)$ & $41,8 \%(n=28)$ & $30,8 \%(n=8)$ \\
\hline Industriegebiet & $6,9 \%(n=5)$ & $10,3 \%(n=15)$ & $14,2 \%(n=24)$ & $25,4 \%(n=17)$ & $7,7 \%(n=2)$ \\
\hline Kneipenviertel & $27,8 \%(n=20)$ & $33,8 \%(n=49)$ & $42,0 \%(n=71)$ & $50,7 \%(n=34)$ & $38,5 \%(n=10)$ \\
\hline Rotlichtviertel & $5,6 \%(n=4)$ & $5,5 \%(n=8)$ & $6,5 \%(n=11)$ & $26,9 \%(n=18)$ & $15,4 \%(n=4)$ \\
\hline Verkehrswege außerorts & $18,1 \%(n=13)$ & $20,7 \%(n=30)$ & $22,5 \%(n=38)$ & $26,9 \%(n=18)$ & $34,6 \%(n=9)$ \\
\hline Großveranstaltungen & $59,7 \%(n=43)$ & $54,5 \%(n=79)$ & $56,2 \%(n=95)$ & $55,2 \%(n=37)$ & $61,5 \%(n=16)$ \\
\hline sonstiges & $12,5 \%(n=9)$ & $8,3 \%(n=12)$ & $9,5 \%(n=16)$ & $10,4 \%(n=7)$ & $7,7 \%(n=2)$ \\
\hline
\end{tabular}

\section{Zeitpunkt der Übergriffe}

Im Hinblick auf eine mögliche Verbindung mit einer Minderung der Hemmung durch bestimmte Umgebungsbedingungen oder den Genuss von Alkohol und Drogen wurde der Zeitpunkt des Geschehens im Tagesverlauf wie auch während des Einsatzes hinterfragt. Deutlich ist, dass sich am Abend und in der Nacht die Übergriffe häufen ( $\triangleright$ Abb. 7).

Schon beim Eintreffen am Notfallort kam es bereits zu Übergriffen, die sich steigerten beim konkreten Kontakt mit dem Patienten. Am häufigsten war der Konflikt bei der Versorgung des
Patienten, was sich sowohl am Einsatzort als auch beim Transport im Rettungsfahrzeug abspielte (vgl. > Tab. 6).

\section{Täter}

Die Gewalt - in welcher Form auch immer - ging in erster Linie vom Patienten selbst aus (79,3\%). Angehörige und Freunde kamen gleich häufig als Aggressoren infrage. Wesentlich seltener waren Fremde (Schaulustige und Unbeteiligte) die Täter ( $\bullet$ Abb. 8). Aus der Tatsache, dass sich bei Einsätzen mehrere Personen als Täter betätigt haben oder mehrere Einsätze mit Über-

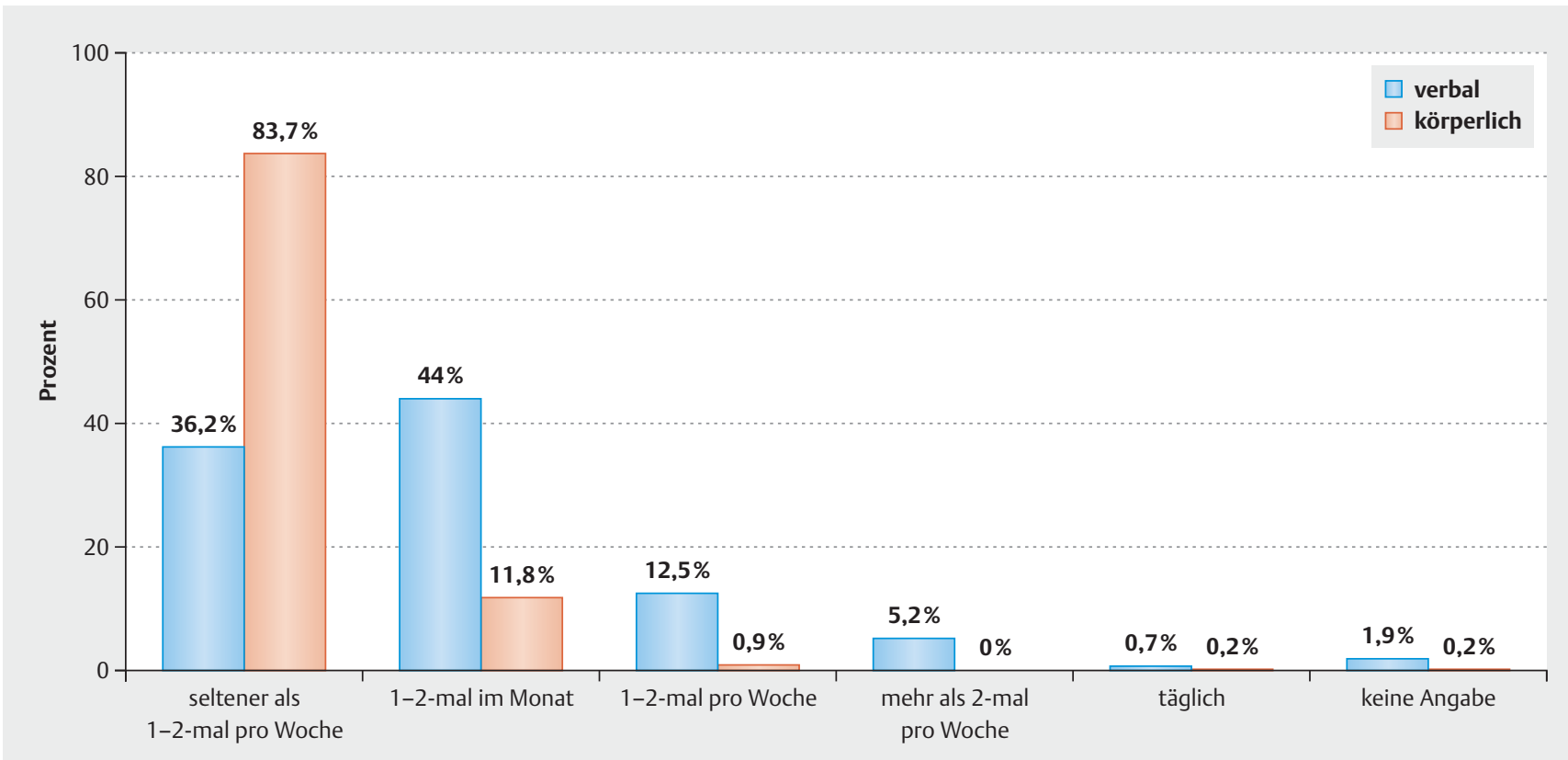

- Abb. 6 Häufigkeit von verbalen und körperlichen Übergriffen. 


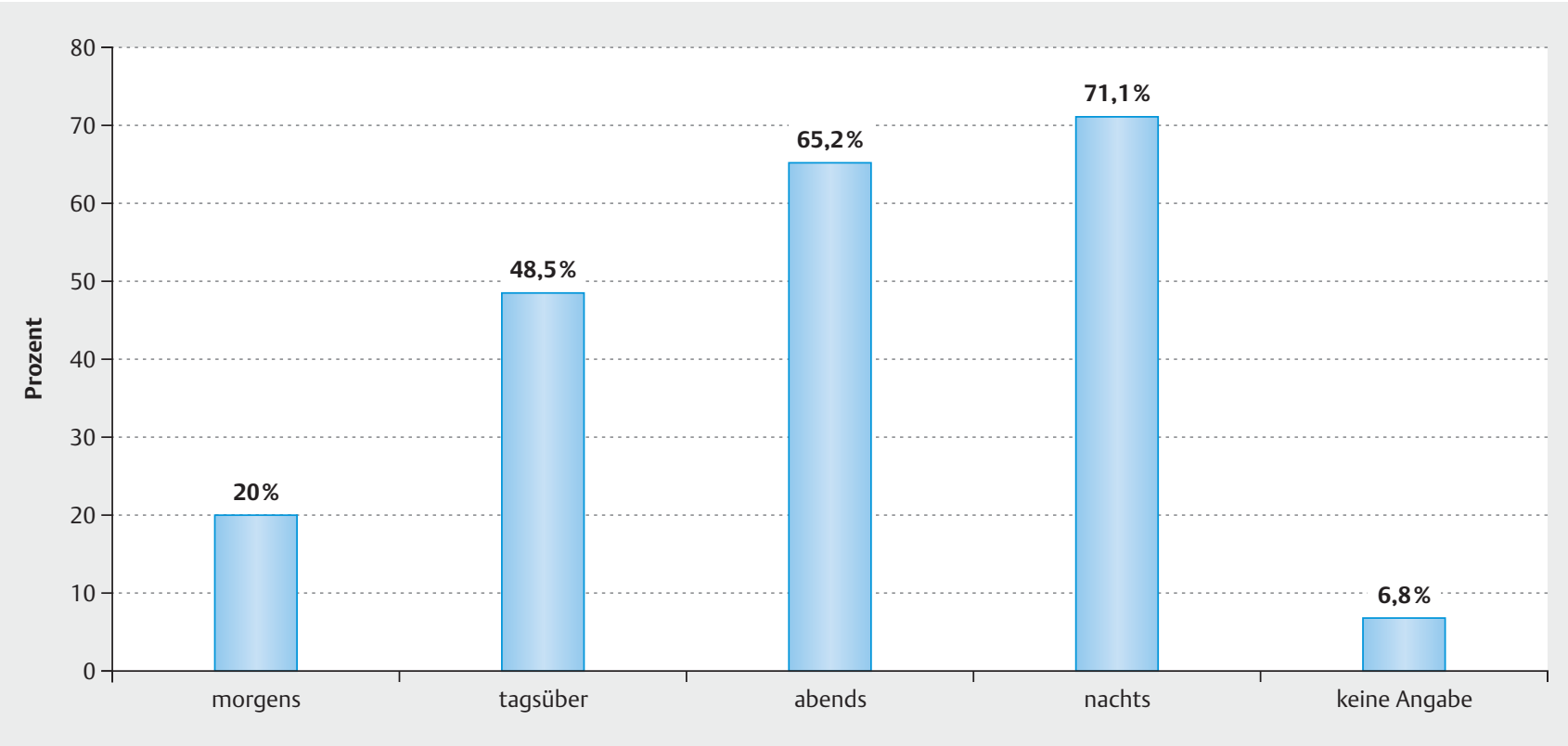

- Abb. 7 Tageszeitpunkte der Übergriffe (Mehrfachnennungen).

griffen zugrunde liegen konnten, resultierten die Mehrfachnennungen.

Dabei waren es fast ausschließlich Männer, die als Täter agierten ( $\bullet$ Abb. 9).

Bei den Ursachen bzw. Auslöser für Gewaltaktivitäten spielen Umgebungsbedingungen eine Rolle. Aus diesem Grund wurde versucht, durch Befragung des Rettungspersonals zu eruieren, inwieweit Alkohol und Drogen bei den Übergriffen im Spiel waren. In über $80 \%$ der Fälle spielten diese beiden Substanzen bei den Aggressionen eine Rolle $(57,4 \%)$ ( $\triangleright$ Abb. 10).

\section{Schäden durch die Gewaltanwendung}

Die Gewalt im Rettungsdienst richtet sich nicht nur gegen das Personal, sondern auch gegen materielle Dinge im Sinne einer Sachbeschädigung. Dabei sind die Fahrzeuge und deren Ausstattung, aber auch persönliche Ausstattung des Personals und des Patienten betroffen. Am häufigsten richtet sich allerdings die Gewalt gegen die Fahrzeuge des Rettungsdienstes ( $\triangleright$ Tab. 7). Der Anteil an den gesamt berichteten Fällen betrug 11,3\% (= 48-mal). Die Häufigkeit der Sachbeschädigungen belief sich meist auf 1-2mal (58,3\%).

- Tab. 6 Zeitpunkt der Übergriffe während des Einsatzes (Mehrfachnennungen).

\begin{tabular}{|c|c|}
\hline & Fälle mit Übergriffen $(n=425)$ \\
\hline beim Eintreffen & $42,4 \%(n=180)$ \\
\hline während der Diagnose & $32,5 \%(n=138)$ \\
\hline während der Behandlung & $56,7 \%(n=241)$ \\
\hline beim Transport & $40,7 \%(n=173)$ \\
\hline Tod des Patienten & $3,3 \%(n=14)$ \\
\hline sonstiges & $5,6 \%(n=24)$ \\
\hline - Krankenhaus (Ankunft/Übergabe) & $2,1 \%(n=9)$ \\
\hline - als kein Patient dabei war (z. B. auf Anfahrt, während Sanitätswachdienst, beim Essen holen) & $1,6 \%(n=7)$ \\
\hline - während Patientengespräch & $0,5 \%(n=2)$ \\
\hline " beim Umlagern & $0,5 \%(n=2)$ \\
\hline " während Erstversorgung & $0,2 \%(n=1)$ \\
\hline - telefonisch & $0,2 \%(n=1)$ \\
\hline - keine konkrete Nennung & $0,5 \%(n=2)$ \\
\hline keine Antwort & $23,3 \%(n=99)$ \\
\hline
\end{tabular}




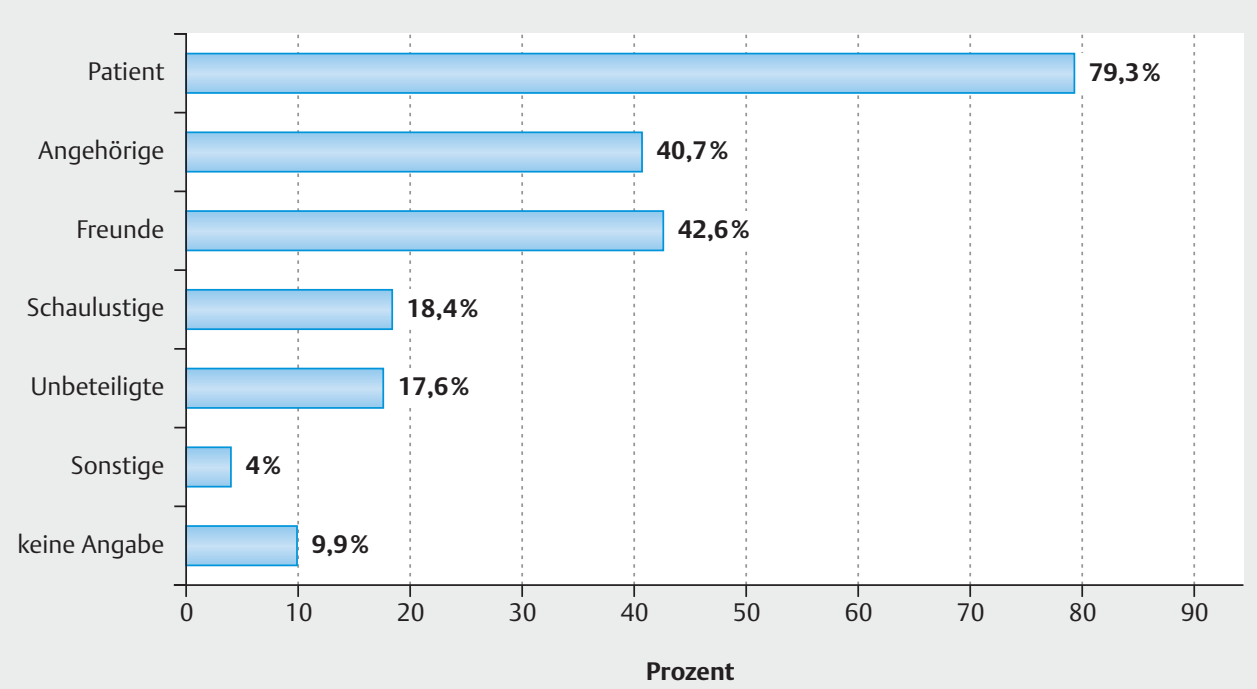

- Abb. 8 Aufschlüsselung der Täter (Mehrfachnennungen).

\section{Auswirkung auf das Personal des Rettungsdienstes}

Bei der Gewalteinwirkung auf Personen - hier Rettungspersonal kommt es nicht nur zu psychischen, sondern auch zu körperlichen (Verletzungs-)Folgen. Diese können dazu führen, dass ein Arzt den Betreffenden krankschreibt und er damit arbeitsunfähig ist, wobei die Länge der Krankschreibung auf die Intensität der Schädigung hinweisen kann. In 97,4\% war keine Krankschreibung erfolgt. Bei 9 Mitarbeitern (2,1\%) war als Folge der Gewaltanwendung eine Krankschreibung notwendig. Die mittlere Dauer der Arbeitsunfähigkeit bei den wenig Betroffenen beträgt 13,5 Tage ( Tab. 8).

\section{Einbeziehung der Polizei}

Nachdem es sich bei der Gewalt gegen Rettungspersonal um eine Straftat handelt, die eine Strafverfolgung entsprechend Strafgesetzbuch (§114) zur Folge haben kann, stellt sich die Frage nach dem Anzeigeverhalten der Betroffenen nach einem erlebten Übergriff.
In 338 Fällen (79,5\%) wurde aus verschiedenen Gründen auf eine Anzeige verzichtet. Die Gründe gehen aus der $>$ Tab. 9 hervor.

Ersichtlich wird, dass sowohl der Zustand des Patienten ein Grund zum Verzicht war, aber auch Erkenntnisse des Personals, dass eine Anzeige zu keinem Erfolg führen würde. Nicht in jedem Fall wurde der Verzicht begründet.

\section{Wünsche für die Zukunft}

56,2\% (239 Mitarbeiter) sahen sich nicht ausreichend auf die neue Situation im Rettungsdienst vorbereitet. Nur 32,7\% (139 Mitarbeiter) glaubten, im Rahmen der Aus- und Fortbildung auf die besondere Situation einer Gewaltanwendung während ihrer Tätigkeit im Rettungsdienst vorbereitet zu sein. Hieraus resultiert der Wunsch von 76\% der Befragten nach mehr Fortbildung und Vorbereitung auf das Thema: Umgang mit Konfliktsituationen.

50 Betroffene haben Wünsche für die Zukunft formuliert bzw. Feststellungen bezüglich des gesamten Themas getroffen ( $\triangleright$ Tab. 10).

- Tab. 7 Art der Sachbeschädigung (Mehrfachnennungen).

\begin{tabular}{|l|l|}
\hline Art der Schädigung & Fälle Sachbeschädigung (n=48) \\
\hline Fahrzeug/Einsatzmittel/RTW & $52,1 \%(n=25)$ \\
\hline Zerstörung persönl. Gegenstände der Einsatzkraft & $16,7 \%(n=8)$ \\
\hline Beschädigung Trage RTW & $8,3 \%(n=4)$ \\
\hline med./techn. Ausstattung & $6,3 \%(n=3)$ \\
\hline Ausrüstung RTW allgemein & $4,2 \%(n=2)$ \\
\hline Hausrat des Patienten & $4,2 \%(n=2)$ \\
\hline sonstiges & $8,3 \%(n=4)$ \\
\hline keine konkrete Nennung & $4,2 \%(n=2)$ \\
\hline
\end{tabular}




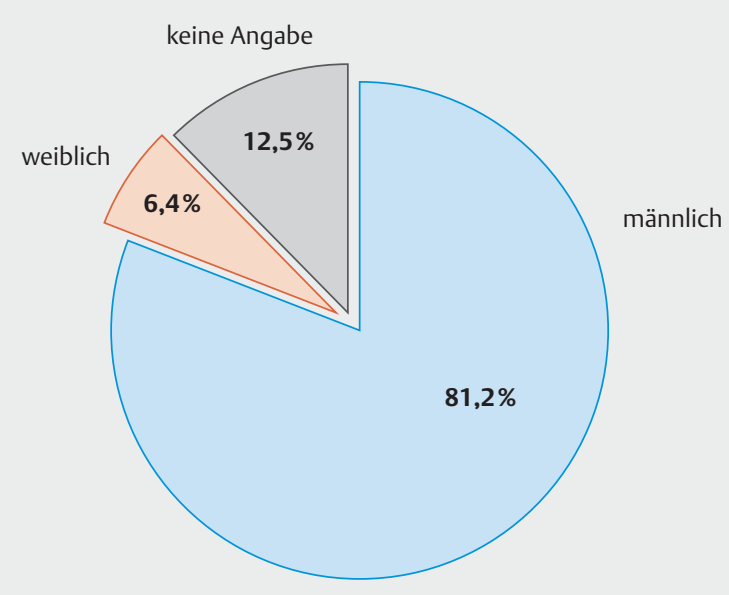

> Abb. 9 Geschlecht der Täter.

\section{Diskussion}

Der Rettungsdienst hat auf der Basis der Landesrettungsdienstgesetze die Aufgabe, die Versorgung von Notfallpatienten am Notfallort und den Notfalltransport durchzuführen und kranke, verletzte und hilfsbedürftige Personen zu versorgen und zu transportieren (siehe beispielhaft BayRDG Art.2). Damit ist das gesamte gesundheitliche Versorgungssystem auf die Hilfeleistung unter Mitwirkung des Rettungsdienstes abgestimmt. Die Einsatzkräfte verstehen sich als Angehörige eines organisierten Hilfeleistungssystem, das Hilfe unterschiedlichster Art gewährt. Zur Umsetzung dieser Hilfe bedienen sie sich der Fahrzeuge des Rettungsdienstes, die sich in der Öffentlichkeit durch ihre besondere Farbgebung kenntlich machen. Auffällig ist auch, und dadurch für die Öffentlichkeit erkenntlich, die besondere Einsatzkleidung des Personals. Schon dadurch heben sich das Personal und die Fahrzeuge des Rettungsdienstes vom Üblichem ab. Die Tatsache der besonderen Kennzeichnung durch eine Dienstkleidung kann heute nicht mehr vor einer möglichen Aggression schützen.

- Tab. 8 Dauer der Arbeitsunfähigkeit.

\begin{tabular}{|l|l|}
\hline Anzahl Tage & Arbeitsunfähigkeit ja $(\mathbf{n}=\mathbf{9})$ \\
\hline 1 Tag & $22,2 \%(\mathbf{n = 2})$ \\
\hline 2 Tage & $11,1 \%(n=1)$ \\
\hline 3 Tage & $11,1 \%(n=1)$ \\
\hline 7 Tage & $11,1 \%(n=1)$ \\
\hline 13 Tage & $11,1 \%(n=1)$ \\
\hline 25 Tage & $11,1 \%(n=1)$ \\
\hline 30 Tage & $11,1 \%(n=1)$ \\
\hline 40 Tage & $11,1 \%(n=1)$ \\
\hline
\end{tabular}

> Tab. 9 Gründe für den Verzicht einer Anzeige bei der Polizei.

\begin{tabular}{|l|l|}
\hline Gründe für den Verzicht & keine Anzeige erstattet (n=338) \\
\hline Patient unter Alkohol/Drogen & $15,1 \%(n=51)$ \\
\hline Patient psychisch beeinträchtigt & $7,1 \%(n=24)$ \\
\hline allg. nicht zurechnungsfähig & $3,0 \%(n=10)$ \\
\hline Krankheit des Patienten Grund für Angriff & $0,6 \%(n=2)$ \\
\hline keine Notwendigkeit, belanglos (z. B. nur verbale Gewalt) & $22,5 \%(n=76)$ \\
\hline aussichtslos, Verfahren würde ohnehin eingestellt & $15,7 \%(n=53)$ \\
\hline (Zeit-)Aufwand vs. Nutzen/Verhältnismäßigkeit & $8,6 \%(n=29)$ \\
\hline Deeskalation erfolgreich & $3,0 \%(n=10)$ \\
\hline Polizei war vor Ort und übernahm & $2,4 \%(n=8)$ \\
\hline kein Zugriff auf Täter möglich (z. B. aufgrund von Flucht) & $1,8 \%(n=6)$ \\
\hline sonstiges & $3,6 \%(n=12)$ \\
\hline weiß nicht & $0,3 \%(n=1)$ \\
\hline keine Angabe & $33,4 \%(n=113)$ \\
\hline
\end{tabular}




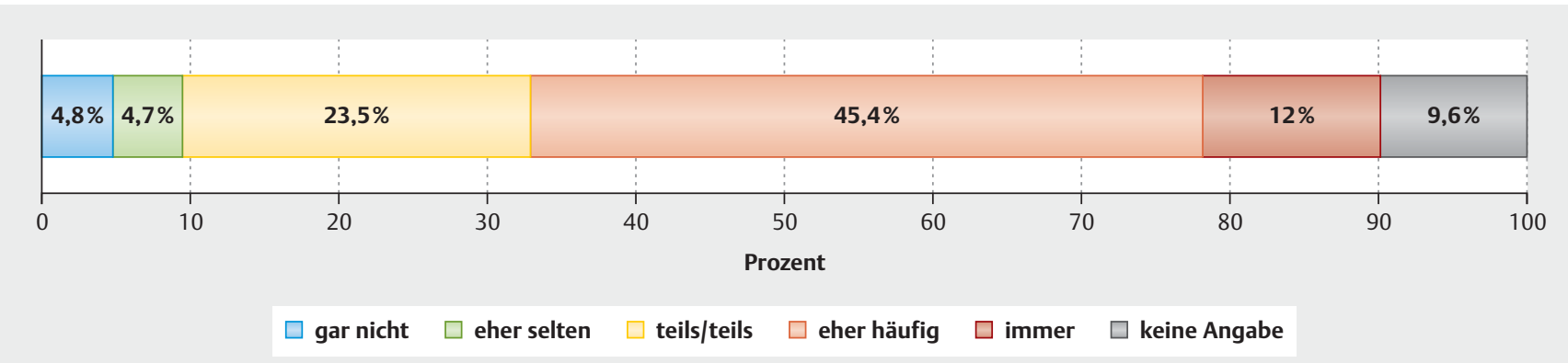

- Abb. 10 Zusammenhang der Gewalteinwirkung mit Alkohol und Drogen beim Täter.

Neben den „normalen“ Einsätzen sog. Regeleinsätze gibt es „problematische“ Einsätze bei besonderen Lagen. Eine der Besonderheiten dieser problematischen Einsätze ist, dass es hierbei zu möglicher Gewaltanwendung kommen kann. Rettungskräfte werden während ihres Einsatzes behindert, angegriffen und erfahren einen respektlosen Umgang. In unsere Analyse gingen ausschließlich Erfahrungen der Rettungskräfte des Deutschen Roten Kreuzes ein und keine Einsatzkräfte aus den Reihen der Feuerwehren. Es konnten 425 Fragebogen in die Analyse aufgenommen werden.
Da der Fragebogen jeweils an die Landesverbände des DRK verteilt wurde, mit der Bitte, diesen an die jeweiligen Rettungsdienste in ihrem Bereich weiterzugeben, war nicht ersichtlich, wie viele Rettungswachen einbezogen werden konnten. Somit ist eine Rücklaufquote nicht ersichtlich. Es sollte nicht die absolute Zahl der Gewalttaten im Rettungsdienst eruiert werden, sondern es handelt sich vielmehr um eine Analyse der Art der Gewaltübergriffe und deren Auswirkungen.

- Tab. 10 Wünsche für die Zukunft (Mehrfachnennungen).

\begin{tabular}{|c|c|}
\hline & Fälle mit Übergriffen $(n=425)$ \\
\hline \multicolumn{2}{|l|}{ Wünsche } \\
\hline Selbstverteidigungskurse/Möglichkeiten zur Selbstverteidigung & $3,3 \%(n=14)$ \\
\hline Deeskalationstrainings & $2,8 \%(n=12)$ \\
\hline angemessene Ausstattung (z. B. Schutzkleidung, Pfefferspray) & $2,1 \%(n=9)$ \\
\hline regelmäßige Fortbildungen durch Fachkräfte (regional, überregional, auch für Ehrenamt) & $1,4 \%(n=6)$ \\
\hline gemeinsame Übungen (auch mit Polizei/Sicherheitskräften) & $1,2 \%(n=5)$ \\
\hline Rechtssicherheit & $0,9 \%(n=4)$ \\
\hline häufigere Mitalarmierung der Polizei, insbesondere bei Alkohol/Drogen/psychische Ursachen & $0,7 \%(n=3)$ \\
\hline schärfere Sanktionen & $0,5 \%(n=2)$ \\
\hline Gefahrenzulagen & $0,2 \%(n=1)$ \\
\hline finanzielle Entschädigung & $0,2 \%(n=1)$ \\
\hline Aufklärung der Bevölkerung über Arbeit des RD & $0,2 \%(n=1)$ \\
\hline Verfahrensanweisungen bezüglich Gewalttaten im Rettungsdienst & $0,2 \%(n=1)$ \\
\hline \multicolumn{2}{|l|}{ Feststellungen } \\
\hline Arbeitgeber unterstützt Fortbildungen nicht & $0,7 \%(n=3)$ \\
\hline es ist keine Ausbildung erfolgt & $0,5 \%(n=2)$ \\
\hline mangelnder Respekt der Bevölkerung gegenüber RD & $0,5 \%(n=2)$ \\
\hline Selbstverteidigungs-/Deeskalationstrainings erzeugen falsche Sicherheit & $0,5 \%(n=2)$ \\
\hline Gewalt im Straßenverkehr nimmt zu & $0,2 \%(n=1)$ \\
\hline Thema Gewalt im RD ist zu aufgebauscht & $0,2 \%(n=1)$ \\
\hline Gewaltbereitschaft steigt & $0,2 \%(n=1)$ \\
\hline Alkoholismus steigt & $0,2 \%(n=1)$ \\
\hline NotSan-Ausbildung in diesem Zusammenhang super & $0,2 \%(n=1)$ \\
\hline keine Angabe & $88,2 \%(n=375)$ \\
\hline
\end{tabular}




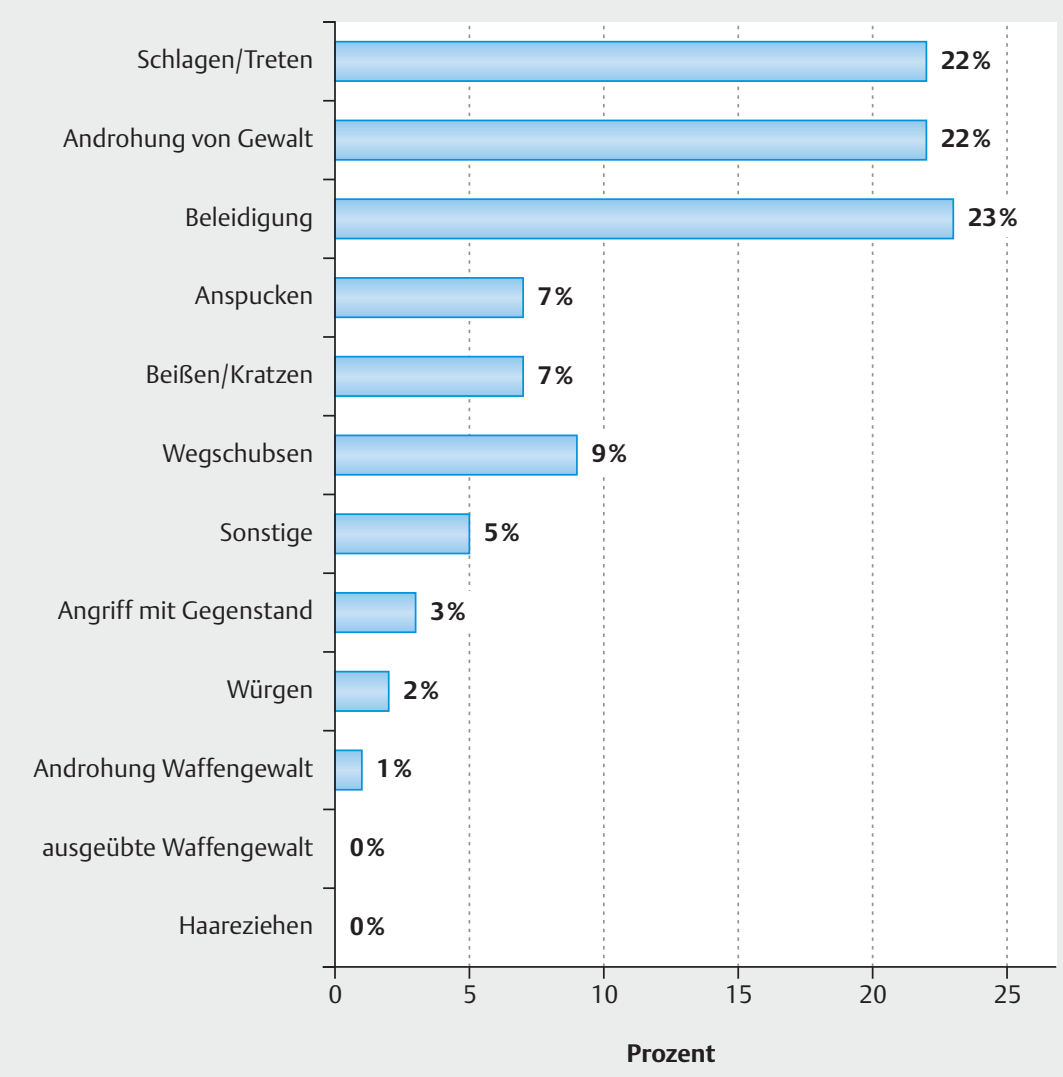

- Abb. 11 Benutzte Mittel der Gewalt - Analyse BRK.

Zum Vergleich wurde eine regionale Auswertung eines großen Bundeslandes des Bayerischen Roten Kreuzes (BRK) einbezogen. Rettungskräfte des BRK gaben für 2019 an, dass Auseinandersetzungen bei Rettungseinsätzen regelmäßig passieren und fast schon an der Tagesordnung seien [7]. In einer Befragung von Mitarbeitern des Malteser Hilfsdienstes wurden Behinderungen und Beleidigungen gleichfalls als Alltagserfahrung beschrieben. Über die $90 \%$ aller Befragten berichten, häufiger (Median: 10) solche Erfahrungen gemacht zu haben [8]. Gewalttätige Übergriffe auf Rettungskräfte gibt es in unterschiedlichen Formen; sie reichen von verbaler Gewalt bis zu tätlichen Übergriffen [9]. In einer Bestandsaufnahme aus 52 Kreisen und kreisfreien Städten in Nordrhein-Westfalen wurde festgestellt, dass fast alle medizinischen Rettungskräfte (98\%) innerhalb eines Jahres schon mindestens einmal verbaler Gewalt sowie Androhung ausgesetzt waren. Opfer eines gewalttätigen Übergriffes wurden mehr als die Hälfte aller Befragten [10]. Im Gegensatz zu einer Auswertung des IKRK, wo seit März 2020 in 13 Ländern 208 Gewalttaten bei Angehörigen des Roten Kreuzes registriert wurden [11], konnten in dieser Umfrage des DRK Erfahrungen von 425 Einsatzkräften aus 12 Monaten in den Jahren 2018/19 dokumentiert werden, wobei Einsatzkräfte durchaus mehrere Übergriffe erlebt haben (vgl. - Tab. 1 und 2). Bei einer Erhebung der Ruhr-Universität Bochum aus dem Jahr 2017 gaben 60 Prozent der befragten Einsatzkräfte an, in den vergangenen 12 Monaten Formen der Gewalt ausgesetzt gewesen zu sein [12]. Bei der Jahresübersicht der Berliner Feuerwehr 2019 konnten 211 strafrechtlich relevante Übergriffe festgestellt werden, das sind 0,05\% der gesamten Einsätze [13]. Bei einer prospektiven Analyse bei 8 ländlichen und 2 städtischen Rettungsdiensten im Jahr 2019 betrug der Anteil der Gewaltanwendungen bei 95 Personen mit 173 Taten 1,2\%. Der Vorteil dieser Studie war, dass es sich um eine einsatzbegleitende Analyse handelte. Bei 42,5\% der Einsätze erfolgte eine Dokumentation, d. h. es konnte jeder 2. Einsatz in die Auswertung einbezogen werden [14]. Allerdings ist zu unterstellen, dass generell die Gewaltanwendungen noch wesentlich höher liegen, weil viele Taten nicht zur Anzeige kommen und eine größere Dunkelziffer besteht.

Bei den einfachen Körperverletzungsdelikten zeigte sich in den Jahren 2012-2017 in der Statistik des Bundeskriminalamtes bei den Mitarbeitern des Rettungsdienstes ein Anstieg von 507 auf 891 Fälle [2], wobei auch hier eine größere Dunkelziffer unterstellt werden darf, da viele derartige Delikte nicht angezeigt werden. Das gleiche gilt für schwere und gefährliche Körperverletzungen mit durchschnittlich 150 Fällen pro Jahr. Betrachtet man die angezeigten Bedrohungen im Verlauf der letzten 6 Jahre, zeigt sich insgesamt ein deutlicher Anstieg [2].

In Übereinstimmung mit der Untersuchung der Universität Bochum geben 425 unsere Mitarbeiter für den Zeitraum von 12 Mo- 


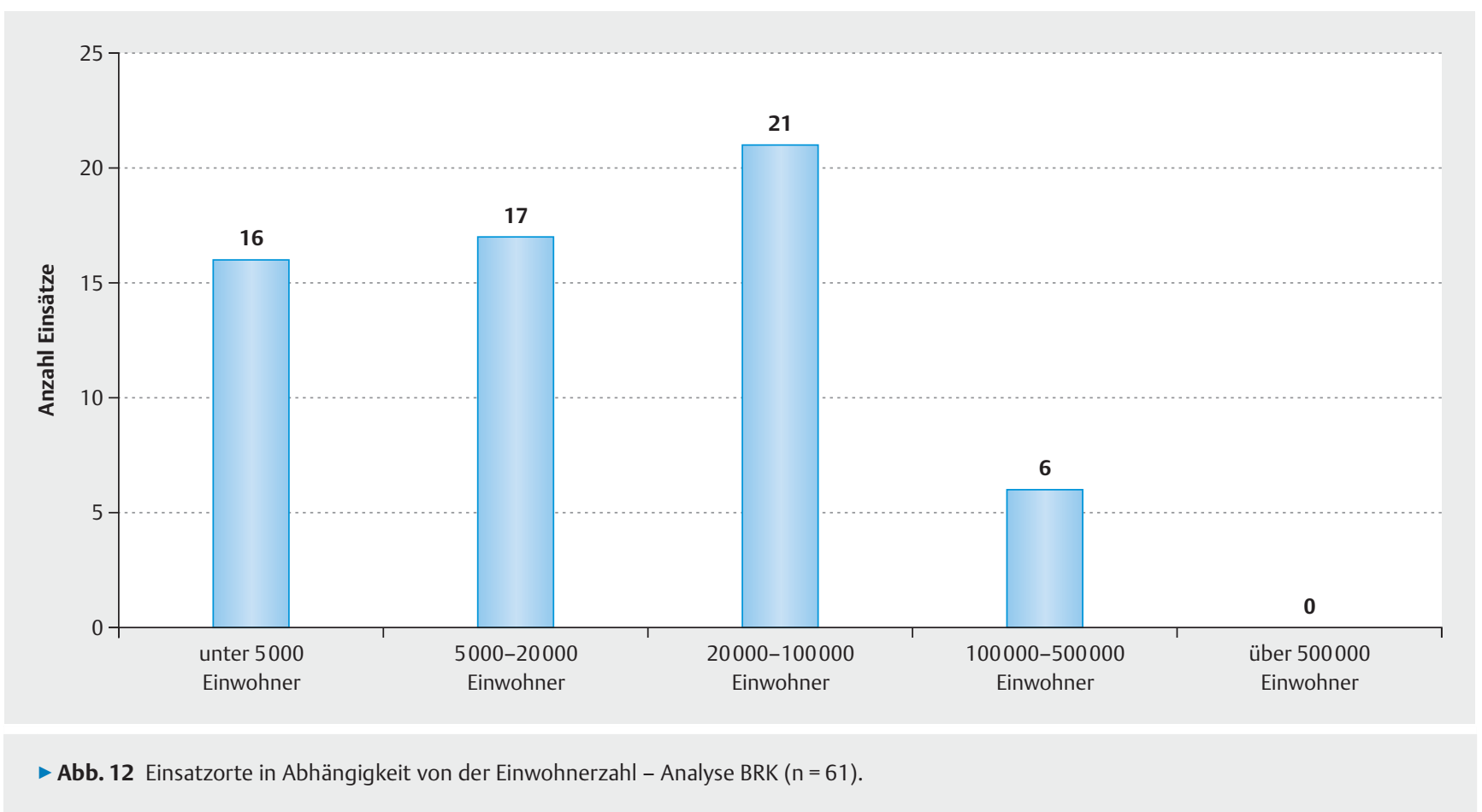

naten bei einer unbekannten Anzahl von Einsätzen an, Opfer von Gewalt geworden zu sein. Feltes und Weigert geben für den Zeitraum von 12 Monaten bei 812 Einsatzkräften in Nordrhein-Westfalen an, dass 64\% der Befragten in 12 Monaten mindestens 1-mal Opfer von Gewalt wurden [9].

Bei der Art der Gewalteinwirkungen zeigt sich, dass verbale Gewalt in Übereinstimmung mit Rau und Leuschner [2] häufiger als solche von körperlicher Gewalt ist. Zwischen 60 und 97,5\% gaben - nach einer Durchsicht der Literatur der Autoren - an, in einem Zeitraum von 12 Monaten verbale Gewalt erfahren zu haben. Auch in der Analyse von Leuchter et al. [14] bei 10 deutschen Rettungsdienstbezirken überwog verbale Gewalt mit 40\% der Fälle, wobei jeweils $25 \%$ nonverbale Gewalt betrafen. In Übereinstimmung mit Feltes und Weigert [9] waren in Nordrhein-Westfalen wie auch bei der vorliegenden DRK-Analyse die Beleidigungen mit 91,1 vs. 90,5\% und Bedrohungen mit 60 vs. 70,6\% die häufigste Art von Übergriffen im Einsatz ( $\bullet$ Abb. 3). Allerdings ist es in einigen Untersuchungen durch die Hinzunahme von Feuerwehrangehörigen zu einer gewissen Verzerrung gekommen. Es ist bekannt, dass Mitarbeiter der Feuerwehren im ausschließlichen Brandeinsatz nicht einmal halb so stark von Gewalt im Einsatz betroffen sind wie Einsatzkräfte in der medizinischen Lebensrettung [9].

Auch der Anteil der körperlichen Gewalt von 14,4\% entspricht den Ergebnissen anderer Untersucher [9]. Bei Leuchter et al. kam es sogar bei $25 \%$ der Mitarbeiter zu einer körperlichen Gewalteinwirkung [14]. Was die Intensität betrifft, waren dies geringfügige bis hin zu lebensgefährlichen Handlungen. Im Vordergrund standen Schlagen und Treten in 32,7\% der Fälle - bei Leuchter 13,5\% - gefolgt von Schubsen (31,5\%) - vs. 26,9\%. In Bayern war Treten und Schlagen die häufigste Form der Gewaltanwen- dung ( $\triangleright$ Abb. 11). Eine Besonderheit scheint das Anspucken zu sein, das bei uns mit einer Häufigkeit von $28 \%$ - vs. 21,2\% in Bayern - zu beobachten war. Allerdings kam es auch vereinzelt zu Gewaltanwendungen, die für den Moment lebensbedrohlich waren, wie Angriffe mit Gegenständen (6,4\%) oder sogar Würgen, was auf eine intensive Aggressivität schließen lässt.

Die eigene Analyse kann Aufschlüsse über die Sensibilität der Mitarbeiter gegenüber von Gewaltereignissen geben. Unterstellt wird dabei, dass Ältere und länger im Rettungsdienst tätige Mitarbeiter nicht nur eine größere Berufserfahrung aufweisen, sondern eher deeskalierend wirken. Inwieweit sich jüngere Mitarbeiter eher provozieren lassen, muss offenbleiben. Dafür könnte sprechen, dass die Rettungskräfte zwischen 18 und 29 Jahren mit einem Anteil von 49,6\%, also fast jeder zweite, und die Älteren ab 50 Jahre und älter nur $8 \%$ ausmachten. Auch hier stimmen die Ergebnisse mit der Untersuchung in Nordrhein-Westfalen überein, die den größten Anteil der Betroffenen bei den bis zu 20 Jahre im Dienst befindlichen hatten und nur ein Anteil von $8,3 \%$ bei den länger Tätigen [9]. Es bleibt offen, ob in der Gruppe der Jüngeren durch selbstbewusstes Auftreten vor Ort (und damit evtl. eine eigene Unsicherheit überspielend) eine überschießende Aktivität resultieren kann, die provozierend wirken könnte. Im Gegenteil zu Älteren, denen durch langjährige Routine und weniger Aufgeregtheit eine gewisse Ruhe unterstellt werden kann, die dazu führt, dass weniger Gewaltanwendungen auftraten oder dass, wenn eine solche Gewalt erfolgte, diese eher akzeptiert und nicht dokumentiert wurde.

Der Anteil der weiblichen Rettungskräfte mit 26,4\% steht im Gegensatz zu der Auswertung in Nordrhein-Westfalen [9], was durch die Tatsache erklärt werden könnte, dass die Rettungskräfte in NRW vor allem aus den Reihen der Feuerwehren kommen, in 


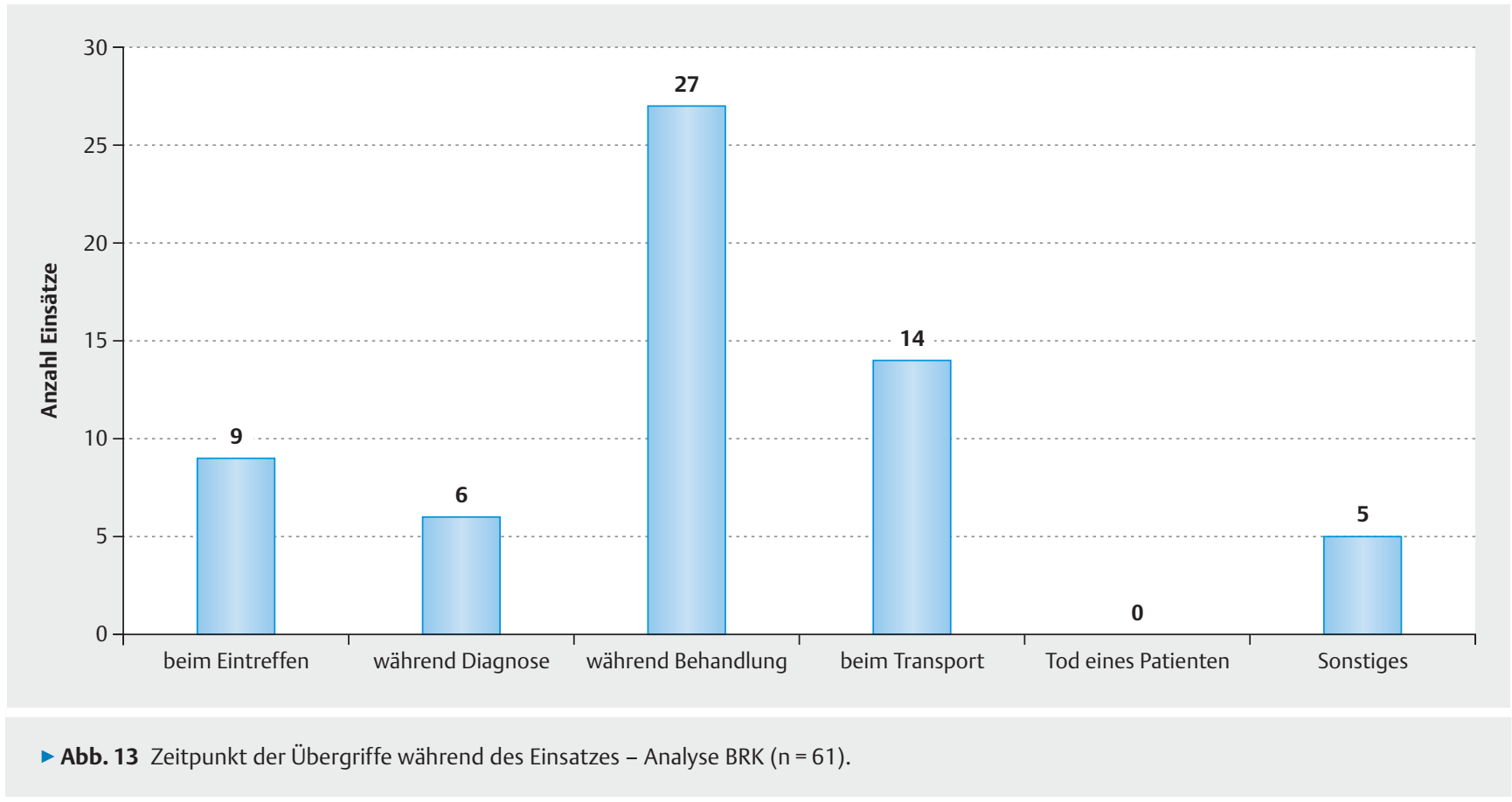

denen weibliche Einsatzkräfte noch seltener sind als im Rettungsdienst des DRK. Hier hat der Anteil in den letzten Jahren kontinuierlich zugenommen. Dieser Anteil kann evtl. auch den Anteil $(13,7 \%)$ von sexuellen Übergriffen erklären, da weibliche Mitarbeiter davon eher betroffen werden als männliche. Bei der Analyse von Leuchter et al. [14] betrug der männliche Anteil der Rettungsfahrzeugbesatzungen mit Gewaltanwendung 1,2\% und der ausschließlich weiblichen $1,7 \%$.

Die berufliche Qualifikation der Betroffenen spiegelt die derzeitige personelle Situation im Rettungsdienst wider. Der hohe Anteil der Rettungssanitäter (33,6\%) resultiert aus dem Mangel von Notfallsanitätern zum Zeitpunkt der Befragung.

Aus der $>$ Tab. 4 wird ersichtlich, dass sich Gewaltanwendungen insbesondere bei Nutzung der Fahrzeuge der Notfallrettung ereigneten. Gerade diese sind durch die Nutzung von Sondersignalen und teilweise einsatzbedingt durch ungünstige Abstellposition am Einsatzort Anlass zu Übergriffen und Abwehrverhalten der Bürger, die sich bis zu Gewaltanwendungen steigern können.

Eine gewisse Absehbarkeit von Gewalt kann evtl. von der Verortung des Einsatzortes abgeleitet werden. Das betrifft sowohl die Unterscheidung in ländliche oder städtische Einsatzorte als auch Einsätze an sozialen Brennpunkten. Bei der isolierten Betrachtung der Verortung auf der Basis der Einwohnerzahl des Einsatzortes, zeigt sich, dass ländliche Regionen keineswegs ausgeschlossen waren und andererseits kein Überwiegen der Großstädte festzustellen war. Im Gegensatz zu Feltes/Weigert [9] liegen unsere räumlichen Schwerpunkte mehr bei Orten mit niedrigen Einwohnerzahlen, z. B. bei unter 5000 Einwohner mit 16,9 versus 3,5\% bei der Auswertung in Nordrhein-Westfalen oder bei den großen Großstädten bei uns mit 6,1 versus 39,3\%. Von einem Schwerpunkt in den großen Großstädten (über 500000 ) kann in unserer Analyse nicht gesprochen werden bei einem Anteil von
6,1\% im Gegensatz zu 39,3\%. Auch in Bayern waren die großen Großstädte erst an 4. Stelle bei der Verortung der Gewaltanwendungen ( $\triangleright$ Abb. 12).

Davon abzugrenzen sind die sozialräumlichen Aspekte, wie z. B. Wohngebiete oder soziale Brennpunkte. Die meisten Einsatzorte, bei denen es zu Gewaltanwendungen kam, waren über die Verortung hinweg im Innerortsbereich, wobei nicht unterschieden wurde, um welche Größenordnung gemäß Einwohnerzahl es ging. Gefolgt wurden diese Orte von Einsätzen in Wohngegenden. Dabei waren es vor allem sozial problematische und bürgerliche Wohnbereiche. Nicht in jedem Fall muss deshalb davon ausgegangen werden, dass eine Gewalt gegen Rettungskräfte in sozial schwachen Wohngebieten ansteht, sondern dies sehr wohl auch in gut situierten Wohngegenden der Fall sein kann. In absteigender Häufigkeit folgten dann Großveranstaltungen und die typischen sozialen Brennpunkte wie Kneipen und Hauptbahnhof.

Eines haben sowohl die ländlichen als auch die städtischen Regionen gemeinsam, das sind die Großveranstaltungen als Ort der Gewaltübergriffe. Eine weitere Gemeinsamkeit ist, dass nachts die Gewaltangriffe am häufigsten sind.

Es sind zu allen Zeitpunkten vom Eintreffen des Rettungsdienstes vor Ort bis zum Abtransport Gewaltausbrüche möglich, am häufigsten geschah dies während der Behandlung (56,7\%), aber auch bereits beim Eintreffen am Notfallort $(42,4 \%)$ und auch während des Transportes (40,7\%). Diese Verteilung stimmt mit den Ergebnissen aus Bayern überein ( $\vee$ Abb. 13 ), sodass gerade während dieser Phase des Einsatzes eine besondere Vorsicht geboten ist.

Erstaunlich war, dass am häufigsten die Gewalt direkt vom Patienten (79,3\%) ausging. In einem direkten Verhältnis zum Patienten standen Angehörige (40,7\%) und Freunde (42,6\%). Aber auch vollkommen Unbeteiligte $(17,6 \%)$ und Schaulustige $(18,4 \%)$ be- 


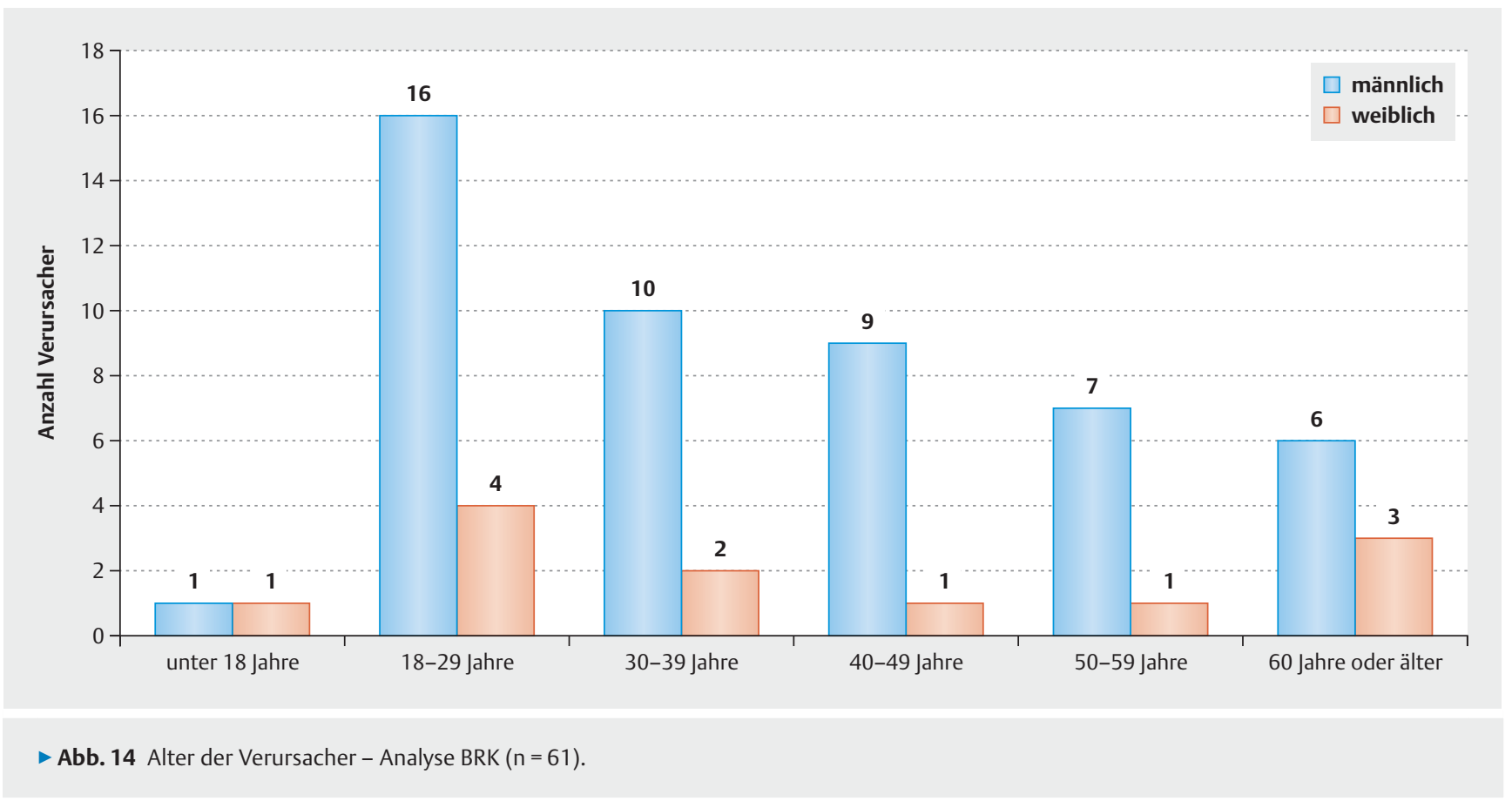

teiligten sich an Gewaltanwendungen, wobei sich selbst mehrere Personen bei einem Einsatz beteiligten.

Die Tatsache, dass der Patient durch die Aktivitäten der Rettungskräfte eventuell sogar unvorbereitet „malträtiert“ wird durch schmerzhafte Maßnahmen (Zugang, Immobilisation) oder aber in seiner Bewegungsfreiheit eingeschränkt wird (Festschnallen auf der Trage), kann eine gewisse Aggressivität auslösen. Auch der Stresslevel durch die Notfallsituation kann ein möglicher Faktor sein, der zu Gewaltausbrüchen führen kann. Denkbar ist aber auch ein inadäquates Verhalten des Rettungspersonals, das gleichfalls unter psychischer Anspannung steht, wobei es zu Überreaktionen kommen kann.

Männliche Täter spielten eine größere Rolle als weibliche ( $\bullet$ Abb. 9), wie auch Scheunpflug und Klewer in ihrer Auswertung 2011 in Sachsen feststellten - Alter zwischen 21 und 40 Jahre, $>50 \%$ alkoholisiert [15]. In der Analyse von Schmidt und Feltes waren 85,4\% der Täter männlich und zwischen 20 und 39 Jahre alt [10]. In Bayern wurde nicht nur das Alter der Verursacher berücksichtigt, sondern auch das Geschlecht. Insgesamt ist der weibliche Tätertyp seltener und nur bei solchen über 60 Jahre im Verhältnis $1 / 3$ zu $2 / 3$ ( Abb. 14).

Am häufigsten in unserer Betrachtung ging die Gewalt von den Patienten selbst aus (79,3\%). In der Analyse von Leuchter et al. [14] waren gleichfalls in 77,9\% der Fälle die Patienten die Täter. Auch in anderen Untersuchungen ist dieser Tätertyp am häufigsten $[9,15]$. Unterstellt werden kann dies auch als Abwehrreaktion auf diagnostische oder therapeutische Maßnahmen [14]. Passanten und andere Verkehrsteilnehmer waren weniger beteiligt $(12,6 \%)$.

Häufig sind Umgebungsbedingungen mit ursächlich für eine Gewaltbereitschaft, dazu gehören vordergründig Alkohol und Drogen. Im Gegensatz zu Schmidt und Feltes [10] konnte keine Differenzierung der Art der Drogen (harte/weiche/Medikamente) vorgenommen werden, sondern die Angaben beruhten ausschließlich auf der subjektiven Beurteilung der Betroffenen. Übereinstimmung besteht aufgrund besserer Beurteilungsfähigkeit bei dem Anteil der unter Alkohol stehenden Täter, wobei ein Anteil von Alkohol bei Schmidt u. Feltes [10] mit 48,9\% angegeben wurde. Auch Leuchter et al. [14] gehen von einem Anteil von 49,1\% bei Alkohol und Drogen aus. Auch an der Schnittstelle des Rettungsdienstes zur Klinik in der Notaufnahme als Ziel des rettungsdienstlichen Transportes wurden in einer Studie der Hochschule Fulda in 85,5\% der Patienten als Auslöser von Gewalt Alkohol und Drogen festgestellt [16]. Es ist keine Frage, dass ein enger Zusammenhang zwischen Alkohol und Drogen und Gewaltbereitschaft besteht. Dies zeigt sich auch darin, dass es an Lokalitäten mit einem höheren Alkohol- und Drogenkonsum (z. B. Hauptbahnhof, Kneipen o.ä.) bei Einsätzen zu Angriffen auf Rettungspersonal kommt. Beide Substanzen senken den Interventionsspiegel und führen bei geringfügigen Dissonanzen zu Aggressivität und Gewalt.

Die teilweise unkontrollierte Gewalt trifft keineswegs nur das Rettungspersonal, sondern auch Sachwerte (11,3\%). Wie die vorliegende Analyse ( $\bullet$ Tab. 7) zeigt, sind dabei alle Bereiche der Rettungsfahrzeuge und deren Inhalt betroffen. Nicht nur, dass es dabei zu Schäden und Zerstörung kommt, sondern auch zu Diebstählen. Bei den Kosten der Ausstattung des Rettungsdienstes resultiert damit auch ein teilweise erheblicher wirtschaftlicher Schaden.

Betrachtet man das Alter der Geschädigten, so sind vordergründig männliche Mitarbeiter im Alter von 40 bis 49 Jahre betroffen. Lediglich bei den jüngeren Mitarbeitern im Alter zwischen 18 und 29 Jahren überwiegt der Anteil der weiblichen Mitarbeiter, was eventuell mit sexueller Gewalt zusammenhängen könnte $(\triangleright$ Abb. 15). 


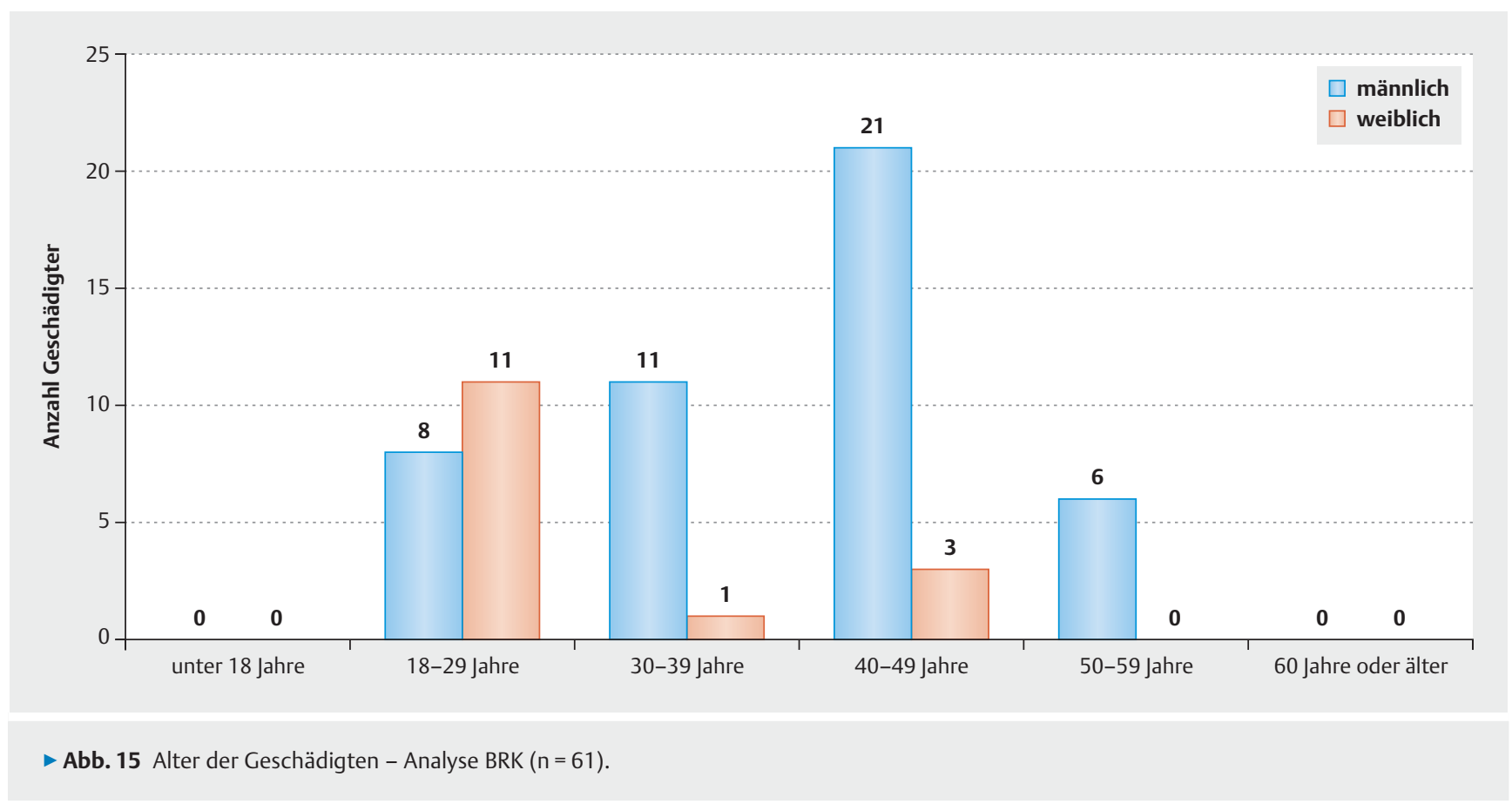

Zu beachten sind die verschiedenen Intensitäten der Übergriffe, die von lediglich banalen (verbalen) Angriffen bis hin zu verschiedenen Verletzungen führen können. Beide können zu einer Beeinträchtigung führen, die eine weitere Teilnahme am Dienst verhindert. In der Folge werden nach einer ärztlichen Konsultation eine Krankschreibung und die Feststellung der Arbeitsunfähigkeit erfolgen. Bei 2,1\% der Betroffenen war die Folge der Gewaltanwendung eine Arbeitsunfähigkeit, die zwischen 1 und 40 Tagen betrug. Hieraus wird auch der wirtschaftliche Schaden beim Betrieb des Rettungsdienstes offenbar.

Nachdem das Phänomen der Gewalt bei Einsatzkräften auch in der Politik angekommen ist, hatte dies zur Folge, dass zum Schutz des Personals im Jahr 2011 der Strafrahmen bei Widerstand gegen Vollstreckungsbeamte von 2 auf 3 Jahre erhöht wurde. Dies betraf jedoch noch nicht das Rettungspersonal. Erst 6 Jahre später kam es zu einer weiteren Verschärfung des § 114 StGB, der nun auch Rettungskräfte einschloss [12]. Ein Verstoß gegen diesen Paragrafen ist somit ein Delikt, das polizeilich verfolgt werden kann/ muss. Auch wenn in den meisten Fällen (79,5\%) auf eine Anzeige und damit die Hinzuziehung der Polizei verzichtet wurde, kam es doch in 19,5\% zu einer Anzeige. Vor diesem Hintergrund und der gesetzlichen Möglichkeit erhebt sich die Frage, warum auf eine polizeiliche Mitwirkung verzichtet wurde. Viele Mitarbeiter begründen dies damit, dass sich an der Situation nichts ändert.

Gründe dafür, warum auf eine Anzeige verzichtet wurde ( $\triangleright$ Tab. 9), lagen sowohl in der geringen Beeinträchtigung oder als auch in der fehlenden Zugriffsmöglichkeit auf den Täter. Auch in Bayern wurde in zwei Drittel der Fälle keine Anzeige erstattet. Die Einsatzkräfte sind aufgrund ihrer bisherigen Erfahrungen enttäuscht, dass viele Verfahren, sofern eine Anzeige erfolgt war, von den Gerichten eingestellt werden.
Nicht außer Acht gelassen dürfen die Folgen eines Übergriffes beim Rettungspersonal. Heinemann [8] beschreibt als längerfristige Folgen einerseits erhöhte Vorsicht, aber auch Verunsicherung, psychosomatische Reaktionen (Ängste, Schweißausbrüche, Schlaflosigkeit) und wiederholte Erinnerungen. Wenn auch die absolute Zahl dieser Reaktionen gering ist, so sind sie doch für die Betroffenen schwerwiegend.

Bei den Wünschen für die Zukunft haben sich nur 50 Betroffene geäußert. Der häufigste Wunsch war in Übereinstimmung mit der Untersuchung von Heinemann [8] eine Schulung in Selbstverteidigung und ein Deeskalationstraining, nachdem dies weder in der Ausbildung vermittelt wurde noch seitens des Arbeitgebers unterstützt wurde ( $>$ Tab. 10). In einer Untersuchung zum Stellenwert eines Deeskalationstrainings in Berlin werden von den Mitarbeitern einer Notaufnahme nach Absolvierung eines Deeskalationstrainings die Fähigkeiten als „hoch“ bis „mittel“ eingeschätzt [17]. Lee stellte dagegen fest, dass die notwendigen Fähigkeiten zum Umgang mit aggressiven Verhaltensweisen trotz der Teilnahme an Deeskalationsprogrammen scheinbar fehlten [18].

In einer Auswertung zum Thema Gewalt in der Notfallmedizin stellten Hofmann und Hachenberg [1] fest, dass gemittelt von den ausgewählten Studien 70\% der Betroffenen sich professionelle Schulungen zu Deeskalation, Eigensicherung und Selbstverteidigung wünschen. Gegen bewusstseinsveränderte Patienten können allerdings Deeskalationstrainings kaum schützen, hier sind jedoch Schulungen in Selbstverteidigungstechniken angezeigt [8]. Auch setzt ein Versuch der Deeskalation voraus, dass der Betreffende in der Lage sein muss, das Gesagte zu verstehen, was bei unter Alkohol und Drogen Stehenden zweifelhaft ist. Weitere Wünsche betreffen einen intensiveren Schutz durch Polizei und schärfere Sanktionen gegenüber den Tätern. Vonseiten der Politik 
wird gefordert, dass die Täter deutlich schneller die Folgen der Taten zu spüren bekommen sollten durch beschleunigte Verfahren, um durch eine schnelle Sanktionierung zu zeigen, dass der Rechtsstaat Straftaten nicht toleriere, sondern entschieden bestrafe [1].

Wenn es um die Ursachen der Gewalt im Rettungsdienst geht, ist seitens der Patienten in zunehmendem Maße ein teilweise überzogener Anspruch gegenüber dem Rettungspersonal festzustellen. Ein Anspruchsdenken hat es schon immer gegeben, aber es wurde nicht versucht, es mit Gewalt durchzusetzen. Im Mittelpunkt steht der „Ich-Bezug“ ohne Rücksicht auf die Solidargemeinschaft. Generell darf aber auch ein Werteverfall in unserer Gesellschaft festgestellt werden, ergänzt durch einen Respektverlust gegenüber Vertretern der staatlichen Institutionen, wobei der Rettungsdienst heute aufgrund seiner Präsentation in der Öffentlichkeit als Teil des Staates eingestuft wird. Gesunken ist die Hemmschwelle, Gewalt anzuwenden und die Achtung voreinander. Feltes und Weigert [9] sehen in ihrer Forschungsarbeit eine Tendenz zur Verrohung und „einen Verlust an Empathie“ in der Gesellschaft, wobei beides von einer zunehmend aggressiven Debatte in der Öffentlichkeit gefördert wird. Dies betrifft nicht nur die Großstädte, sondern findet sich auch in ländlichen Regionen. Inwieweit auch den Medien eine Mitschuld gegeben werden kann, wenn in entsprechenden Sendungen dazu aufgefordert wird, sich „nichts gefallen zu lassen“ und dabei zu sensibilisieren im Hinblick auf Widerstand, kann weder erkannt noch belegt werden [20].

Mögliche Schutzmechanismen sind einmal der Rückzug und der Verzicht der Hilfeleistung, wenn eine Gewaltsituation erkennbar ist. Gerade jüngere Mitarbeiter im Rettungsdienst müssen auf die mögliche Gewalt hingewiesen und geschult werden. Schwerpunkt einer Fortbildung sollte die Verbesserung der Kommunikation und die Deeskalation sein. Nur ein letztes Mittel kann eine gezielte Schulung in Selbstverteidigung sein. Über den generellen Einsatz von Schutzwesten gehen die Meinungen auseinander [4]. In verschiedenen Studien sprach sich die Mehrheit der Rettungskräfte gegen das Tragen von Schutzwesten aus [1]. Auch eine Bewaffnung scheint kein realer Weg zu sein und ist deshalb abzulehnen.

\section{Limitationen}

Eine grundsätzliche Einschränkung liegt in der retrospektiven Befragung mittels Fragebogen. Die Teilnehmer nahmen freiwillig an der Befragung teil, wobei keine Repräsentanz gewährleistet war. Eine Überprüfung der Ergebnisse konnte nicht stattfinden, da die Beantwortung anonym war. Die Zahl der Übergriffe konnte nicht in Relation zur Gesamtzahl der Einsätze an den jeweiligen Standorten gesetzt werden, sondern bezieht sich lediglich auf die gemeldeten Fallzahlen. Es kann nicht ausgeschlossen werden, dass es Antwortverzerrungen durch die Fragebogenkonstruktion gekommen sein könnte.

Es darf unterstellt werden, dass nicht alle insbesondere niederschwelligen Übergriffe dokumentiert wurden und deshalb wahrscheinlich eine größere Dunkelziffer besteht. Da nur diejenigen, die definitiv eine Gewalt erfahren haben, den Fragebogen ausgefüllt haben, kann es andererseits zu einer Überschätzung der Gewalterfahrungen kommen.

\section{Fazit}

Auch wenn die Analyse nicht repräsentativ sein kann, so sind doch folgende Konsequenzen zu ziehen und nachfolgende Forderungen zu erheben:

- Schulung des Personals im Rettungsdienst im Hinblick auf Deeskalation, Kommunikation und Selbstschutz

- Schaffung eines Angebots zur Hilfe nach psychisch traumatisierten Einsätzen

- Identifikation von Einsatzlagen, von denen Gewaltanwendungen ausgehen könnten (z. B. Festivitäten mit vermehrtem Alkoholgenuss u.a.) einschließlich der Fähigkeiten zur Früherkennung potenzieller Aggressions- und Gewaltsituationen

- frühzeitige Einbeziehung der Polizei bei verdächtigen Meldebildern durch die Leitstelle

- Dokumentation stattgehabter Gewalt und konsequente Weiterleitung an die Strafverfolgungsbehörden

- Schaffung einer verbindlichen Erfassung beim Arbeitgeber/ Dienstherrn und Nachbereitung für Gewaltanwendungen auf der Basis einer standardisierten Meldeverfahrens

Interessenkonflikt

Die Autorinnen/Autoren geben an, dass kein Interessenkonflikt besteht.

Literatur

[1] Hofmann T, Hachenberg T. Gewalt in der Notfallmedizin - gegenwärtiger Stand in Deutschland. Anästhesiol Intensivmed Notfallmed Schmerzther 2019; 54: 145-154

[2] Rau M, Leuschner F. Gewalterfahrungen von Rettungskräften im Einsatz - Eine Bestandsaufnahme der empirischen Erkenntnisse in Deutschland. Neue Kriminalpolitik (NK) 2018; 30: 316-335

[3] [Anonym]. Angriffe auf Rettungsdienste auf Rekordniveau. Im Internet (Stand: 03.09.2020): https://www.aerzteblatt.de/nachrichten/116018/ Angriffe-auf-Rettungsdienste-auf-Rekordniveau

[4] DRK Landesverband Westfalen-Lippe. Merkblatt Handlungshilfe „Gewalt gegen Einsatzkräfte“. Im Internet (Stand: 03.09.2020): https://www. drk-westfalen.de/fileadmin/Eigene_Bilder_und_Videos/Downloads/ Notfallnachsorge/Merkblatt_Handlungshilfe_Gewalt_gegen_EK_kurz. pdf

[5] Schwind HD. Kriminologie: Eine praxisorientierte Einführung mit Beispielen. 19. Aufl. Heidelberg: Kriminalistik Verlag; 2009

[6] [Anonym]. Was ist Gewalt und welche Formen gibt es? Im Internet (Stand: 04.06.2020): https://www.juraforum.de/lexikon/gewalt

[7] Bayerisches Rotes Kreuz. Aggression im Rettungsdienst - Stand 01.07.2016, persönl. Mitteilung

[8] Heinemann W. Gewalt im Rettungsdienst: Welche Auswirkungen haben Angriffe bei Einsätzen. Rettungsdienst 2011; 34: 324-327

[9] Feltes T, Weigert M. Abschlussbericht des Forschungsprojektes. Gewalt gegen Einsatzkräfte der Feuerwehren und Rettungsdienste in Nordrhein-Westfalen. Bochum: Ruhr Universität Bochum; 2018

[10] Schmidt J, Feltes T. Gewalt gegen Rettungskräfte. Bestandsaufnahme zur Gewalt gegen Rettungskräfte in Nordrhein-Westfalen. Abschlussbericht Ruhr-Universität Bochum, April 2012. Im Internet (Stand: 11.12.2020): http://www.unfallkasse-nrw.de/fileadmin/Server/ download/PdF_2012/Gewalt_gegen_Rettungskraefte.pdf

[11] Mäurer DK. Mehr Gewalt gegen medizinisches Personal: Im Internet (Stand: 31.05.2020): www.tagesschau.de/ausland/rotes-kreuz-gewalt101.html 
[12] Deutscher Bundestag. Bundestag beschließt besseren Schutz für Beamte und Rettungskräfte. Im Internet (Stand: 11.12.2020): www.bundestag.de/dokumente/textarchiv/2017/kw17-de-schutz-rettungskraefte503660

[13] Berliner Feuerwehr. Jahresbericht 2019. Im Internet (Stand: 28.06.2020): https://www.berliner-feuerwehr.de/fileadmin/bfw/dokumente/Publikationen/Jahresberichte/jahresbericht2019.pdf

[14] Leuchter F, Hergaten T, Heister U et al. Gewalt gegen Rettungsdienstmitarbeiter - eine einsatzbegleitende Analyse in ländlichen und städtischen Rettungsdienstbereichen. Dtsch Arztebl Int 2020; 117: 460-461

[15] Scheunpflug S, Klewer J. Gewaltsituationen im Rettungsdienst. HeilberufeScience 2013; 4: 89-92
[16] Hochschule Fulda. Gewalt in der Notaufnahme. Im Internet (Stand: 11.12.2020): https://www.hs-fulda.de/unsere-hochschule/alle-meldungen/meldungsdetails/detail/gewalt-in-der-notaufnahme

[17] Frick J, Stagmann A, Möckel M et al. Erleben von aggressiven Verhalten in der Notaufnahme nach Etablierung eines Deeskalationstrainings. Notfall Rettmed 2018; 21: 349-356

[18] Lee F. Violence in A\&E: the role of training and self-efficacy. Nurse Stand 2001; 15: 33-38

[19] Wernicke C. Männlich, jung, aggressiv. Im Internet (Stand: 26.09.2020): https://www.sueddeutsche.de/panorama/studie-zu-gewalt-gegeneinsatzkraefte-maennlich-jung-aggresiv-1.3814103?print=true

[20] Merker. Nichts gefallen lassen! Im Internet (Stand: 08.10.2020): https:// www.zeit.de/1958/40/nichts-gefallen-lassen 